\title{
On More General Fractional Differential Equations Involving Mixed Generalized Derivatives with Nonlocal Multipoint and Generalized Fractional Integral Boundary Conditions
}

\author{
Wafa Shammakh, Hadeel Z. Alzumi $\mathbb{D}$, and Bushra A. AlQahtani \\ Mathematics Department, Faculty of Science, University of Jeddah, Saudi Arabia \\ Correspondence should be addressed to Hadeel Z. Alzumi; hzalzumi@uj.edu.sa
}

Received 11 March 2020; Revised 8 May 2020; Accepted 7 October 2020; Published 16 November 2020

Academic Editor: Seppo Hassi

Copyright $\odot 2020$ Wafa Shammakh et al. This is an open access article distributed under the Creative Commons Attribution License, which permits unrestricted use, distribution, and reproduction in any medium, provided the original work is properly cited.

This paper deals with the existence of solutions for a new boundary value problem involving mixed generalized fractional derivatives of Riemann-Liouville and Caputo supplemented with nonlocal multipoint boundary conditions. The existence results for inclusion case are also discussed. The nonlinear term belongs to a general abstract space, and our results rely on modern theorems of fixed point. Ulam stability is also presented. We provide some examples that well-illustrate our main results.

\section{Introduction and Preliminaries}

In this paper, we study a new generalized boundary value problem (GBVP) that involves both generalized RiemannLiouville and Caputo derivatives via nonlocal multipoint and generalized fractional integral boundary conditions given by

$$
\left\{\begin{array}{l}
{ }^{R L} D_{0^{+}}^{\alpha, \rho}\left({ }^{\mathrm{C}} D_{0^{+}}^{\beta} y(t)\right), \quad 0<\alpha, \beta \leq 1, \\
y(0)=\alpha_{1} \sum_{i=1}^{m} \sigma_{i} I_{0^{+}}^{q, \rho} y\left(\varepsilon_{i}\right)+\alpha_{2} \sum_{i=1}^{p} \delta_{i} y\left(\eta_{i}\right), \\
\gamma y(1)=b_{1} \sum_{i=1}^{m} \mu_{i} I_{0^{+}}^{q, \rho} \gamma y\left(\varepsilon_{i}\right)+b_{2} \sum_{i=1}^{p} r_{i} \gamma y\left(\eta_{i}\right),
\end{array}\right.
$$

where the derivatives ${ }^{\mathrm{RL}} D_{0^{+}}^{\alpha, \rho}$ and ${ }^{\mathrm{C}} D_{0^{+}}^{\beta, \rho}$ denote the generalized Riemann-Liouville fractional derivative of order $0<\alpha \leq 1$ and generalized Caputo fractional derivative of order $0<\beta \leq 1$, respectively. $I_{0^{+}}^{q, \rho}$ is the generalized Riemann-Liouville fractional integral of order $0<q<1$, and the nonlinear term $g$ is given in the Orlicz space $L_{\mathscr{G}}[0,1]$.
We also investigate the corresponding inclusion problem which is given by

$$
\left\{\begin{array}{l}
{ }^{R L} D_{0^{+}}^{\alpha, \rho}\left({ }^{C} D_{0^{+}}^{\beta, \rho} y(t)\right) \in G(t, y(t)), \quad 0<\alpha, \alpha \leq 1, \\
y(0)=\alpha_{1} \sum_{i=1}^{m} I^{q, \rho} y\left(\varepsilon_{i}\right)+\alpha_{2} \sum_{i=1}^{p} \delta_{i} y\left(\eta_{i}\right), \\
\gamma y(1)=b_{1} \sum_{i=1}^{m} \mu_{i} I^{q, \rho} \gamma y\left(\varepsilon_{i}\right)+b_{2} \sum_{i=1}^{p} r_{i} \gamma y\left(\eta_{i}\right),
\end{array}\right.
$$

where $a_{i}, b_{i} \in \mathbb{R}(i=1,2) ; \delta_{i}, r_{i} \in \mathbb{R}^{+}(i=1, \cdots, p) ; \sigma_{i}, \mu_{i} \in \mathbb{R}^{+}$ $(i=1, \cdots, m) ; \gamma=t^{1-\rho}(d / d t) ;$ and $\rho>0$, and $G:[0,1] \times \mathbb{R}$ $\longrightarrow \mathscr{P}(\mathbb{R})$ is a multivalued map. $\mathscr{P}(\mathbb{R})$ is the family of all subsets of $\mathbb{R}$.

The concept of generalized fractional derivatives and integrals was introduced by Katugampola in $[1,2]$. This new form of fractional integral operator generalizes Riemann-Liouville and Hadamard fractional derivative into a single form and satisfy a semigroup property. Jarad et al. [3] proposed a new generalized fractional calculus based on a special case of proportional derivatives where the kernel of the fractional operator involves an exponential function. 
This generalization contains Riemann-Liouville and Caputo fractional derivatives and integrals as special cases. Also, there were works on general fractional derivative and integral containing a Mittag-Leffler function. For more new definitions of generalized fractional calculus, one can see [4-7]. These generalizations are helpful to reach a better form of the real models that appeared in various fields such as physics, chemistry, aerodynamics, and electrorheology employing fractional differential equations (see [8-17]). The existence and uniqueness results of fractional boundary value problems involving fractional derivatives and derivative of a generalized type were established by many authors (for example, see [18-21]).

On the other hand, Birnbaum and Orlicz [22] generalized the classical Lebesgue space $L_{p}, 0<p<\infty$, into a general abstract space, which was called Orlicz space. This generalization is based on replacing the function $x^{p}$ with more general function $f$.

In what follows, we recall some basic concepts of fractional calculus $[1,2,4]$ and Orlicz space $[22]$ that are needed throughout the paper.

Consider the space $X_{c}^{p}(a, b)(c \in \mathbb{R}, 1 \leq p \leq \infty)$ of those complex-valued Lebesgue measurable functions $f$ on $[a, b]$ for which $\|f\|_{X_{c}^{p}(a, b)}<\infty$, where the norm is defined by

$$
\|f\|_{X_{c}^{p}}=\left(\int_{a}^{b}\left|t^{c} f(t)\right|^{p} \frac{d t}{t}\right)^{1 / p}<\infty .
$$

For $0 \leq \varepsilon \leq 1$, we denote by $\mathrm{AC}_{\delta}^{n}[a, b]$ the space of all absolutely continuous real-valued functions on $[a, b]$, where

$$
\left\{\mathrm{AC}_{\delta}^{n}[a, b]=f:[a, b] \longrightarrow \mathbb{C} \text { and } \delta^{n-1} f \in \mathrm{AC}[a, b], \delta=t^{1-\rho} \frac{d}{d t}\right\}
$$

endowed with the norm $\|f\|_{C_{\delta}^{n}}=\sum_{k=0}^{n}\left\|\delta^{k} f\right\|_{C}$. Moreover, we introduce

$$
\begin{aligned}
C_{\delta, \varepsilon}^{n}[a, b]= & \left\{:[a, b] \longrightarrow \mathbb{C} \text { and } \delta^{n-1} f \in C[a, b],\right. \\
& \left.\delta^{n} f \in C_{\varepsilon, \rho}[a, b], \delta=t^{1-\rho} \frac{d}{d t}\right\},
\end{aligned}
$$

equipped with the norm $\|f\|_{C_{\delta, \varepsilon}^{n}}=\sum_{k=0}^{n-1}\left\|\delta^{k} f\right\|_{C}+\left\|\delta^{n} f\right\|_{C_{\varepsilon, \rho}}$, where $C_{\varepsilon, \rho}[a, b]=\left\{f:[a, b] \longrightarrow \mathbb{R}:\left(t^{\rho}-a^{\rho}\right)^{\varepsilon} f(t) \in C([a, b]\right.$, $\mathbb{R})\}$ and $C([a, b], \mathbb{R})$ is a Banach space of all continuous functions on $[a, b]$ into $\mathbb{R}$ endowed with the norms $\|f\|_{C_{\varepsilon, \rho}}=$ $\left\|\left(t^{\rho}-a^{\rho}\right)^{\varepsilon} f(t)\right\|_{C}$ and $\|x\|_{C}=\sup \{|x(t)|: t \in[a, b]\}$, respectively. Notice that $C_{\delta, 0}^{n}[a, b]=C_{\delta}^{n}[a, b]$.

Definition 1. For $-\infty<a<t<b<\infty$, the left-sided generalized fractional integral of $f \in X_{c}^{p}(a, b)$ of order $\alpha>0$ and $\rho>0$ is defined by

$$
\left(I_{a_{+}}^{\alpha, \rho} f\right)(t)=\frac{\rho^{1-\alpha}}{\Gamma(\alpha)} \int_{a}^{t} \frac{s^{\rho-1}}{\left(t^{\rho}-s^{\rho}\right)^{1-\alpha}} f(s) d s
$$

Definition 2. For $0 \leq a<x<b<\infty$, the generalized Riemann-Liouville fractional derivative associated with generalized fractional integrals (2) is defined by

$$
\begin{aligned}
\left({ }^{R L} D_{a_{+}}^{\alpha, \rho} f\right)(t) & =\left(t^{1-\rho} \frac{d}{d t}\right)^{n}\left(I_{a_{+}}^{n-\alpha, \rho} f\right)(t) \\
& =\frac{\rho^{\alpha-n+1}}{\Gamma(n-\alpha)}\left(t^{1-\rho} \frac{d}{d t}\right)^{n} \int_{a}^{t} \frac{s^{\rho-1}}{\left(t^{\rho}-s^{\rho}\right)^{\alpha-n+1}} f(s) d s
\end{aligned}
$$

Definition 3. The generalized Caputo-type fractional derivative of $f \in A C_{\delta}^{n}[a, b]$ of order $\alpha \geq 0$ is defined by

$$
\begin{gathered}
\left({ }^{C} D_{a_{+}}^{\alpha, \rho} f\right)(t)=D_{a_{+}}^{\alpha, \rho}\left[f(t)-\sum_{k=0}^{n-1} \frac{\delta^{k} f(a)}{k !}\left(\frac{t^{\rho}-a^{\rho}}{\rho}\right)^{k}\right](x), \\
\delta=x^{1-\rho} \frac{d}{d x},
\end{gathered}
$$

where $n=[\alpha]+1$.

The following shows some important properties which are used in the sequel.

Lemma 4. Let $f \in A C_{\delta}^{n}[a, b]$ or $C_{\delta}^{n}[a, b]$, and $\alpha \in \mathbb{R}$. Then,

$$
I_{a_{+}}^{\alpha, \rho C} D_{a_{+}}^{\alpha, \rho} f(t)=f(t)-\sum_{k=0}^{n-1} \frac{\left(\delta^{k} f\right)(a)}{k !}\left(\frac{t^{\rho}-a^{\rho}}{\rho}\right)^{k} .
$$

In particular, if $0<\alpha \leq 1$, we have

$$
I_{a_{+}}^{\alpha, \rho C} D_{a_{+}}^{\alpha, \rho} f(t)=f(t)-f(a)
$$

Lemma 5. Let $\operatorname{Re}(\alpha)>0, n \leq-[-\operatorname{Re}(\alpha)], f \in L(a, b)$ and $I_{a_{+}}^{\alpha, \rho}$ $f \in A C_{\delta}^{n}[a, b]$. Then,

$$
I_{a_{+}}^{\alpha, \rho R L} D_{a_{+}}^{\alpha, \rho} f(t)=f(t)-\sum_{j=1}^{n-1} \frac{D_{a_{+}}^{\alpha-j, \rho} f(a)}{\Gamma(\alpha-j+1)}\left(\frac{t^{\rho}-a^{\rho}}{\rho}\right)^{\alpha-j} .
$$

In particular, if $0<\alpha \leq 1$, we have

$$
I_{a_{+}}^{\alpha, \rho R L} D_{a_{+}}^{\alpha, \rho} f(t)=f(t)-\frac{D_{a_{+}}^{\alpha-1, \rho} f(a)}{\Gamma(\alpha)}\left(\frac{t^{\rho}-a^{\rho}}{\rho}\right)^{\alpha-1} .
$$

Lemma 6. For a function $f \in X_{c}^{p}(a, b), \rho \geq c$, we have

$$
\left(D_{a_{+}}^{\alpha, \rho}\left(I_{a_{+}}^{\beta, \rho} f\right)\right)(t)=\left(I_{a_{+}}^{\beta-\alpha, \rho} f\right)(t), \quad \beta>\alpha>0,
$$

which holds almost everywhere on $[a, b]$. If $\alpha=\beta$, we have

$$
\left(D_{a_{+}}^{\alpha, \rho}\left(I_{a_{+}}^{\alpha, \rho} f\right)\right)(t)=f(t)
$$

almost everywhere. 
Lemma 7. For $0<\alpha<\beta$, we have

$$
I_{0_{+}}^{\beta, \rho}\left(t^{\rho(\alpha-1)}\right)=\frac{t^{\rho(\alpha+\beta-1)} \Gamma(\alpha)}{\rho^{\beta} \Gamma(\alpha+\beta)} .
$$

Definition 8. Let $\varphi:[0, \infty) \longrightarrow[0, \infty)$ be right continuous, monotone, increasing function with $\varphi(0)=0, \lim _{t \rightarrow \infty} \varphi(t)=$ $\infty$, and $\varphi(t)>0$ whenever $t>0$. Then, the function defined by

$$
\mathscr{G}(x)=\int_{0}^{x} \varphi(t) d t, x \geq 0
$$

is called the $N$-function.

Alternatively, the function $\mathscr{G}$ is an $N$-function iff $\mathscr{G}$ is continuous, even, and convex with $\lim _{x \rightarrow 0}(\mathscr{G}(x) / x)=0$, $\lim _{x \rightarrow \infty} \mathscr{G}(x) / x=\infty$ and $\mathscr{G}(x)>0$ if $x>0$.

Note that a function $\mathscr{G}:[0, \infty) \longrightarrow[0, \infty)$ is called a Young function if it is convex and satisfies the conditions

$$
\begin{gathered}
\mathscr{G}(0)=\lim _{x \longrightarrow 0^{+}} \mathscr{G}(x)=0, \\
\lim _{x \longrightarrow \infty} \mathscr{G}(x)=\infty .
\end{gathered}
$$

For an $N$-function $\mathscr{G}$, we define

$$
\mathscr{G}^{*}(x)=\int_{0}^{x} \varphi^{-1}(t) d t, \quad x \geq 0
$$

where $\varphi^{-1}$ is the right inverse of the right derivative of $\varphi$ of $\mathscr{G}$ and is called the complementary of $\mathscr{G}$ and it satisfies the condition

$$
\mathscr{G}^{*}(x)=\sup \{t x-\mathscr{G}(t): t \geq 0\}, \quad \forall x \geq 0 .
$$

Notice that the function $\mathscr{G}^{*}$ is also an $N$-function and the complementary pairs $\mathscr{G}$ and $\mathscr{G}^{*}$ satisfy the following Young inequality:

$$
x t \leq \mathscr{G}(x)+\mathscr{G}^{*}(t), \quad \forall x, t \geq 0 .
$$

Definition 9. For an $N$-function $\mathscr{G}$, the Orlicz space $L_{\mathscr{G}}$ $([0,1])$ is the space of measurable functions $x:[0,1] \longrightarrow$ $\mathbb{R}$ such that $\int_{0}^{1} \mathscr{G}(|x(t)|) d t<\infty$. This space is endowed with the Luxemburg norm, i.e.,

$$
\|x\|_{\mathscr{G}}=\inf \left\{\lambda>0: \int_{0}^{1} \mathscr{G}\left(\frac{|x(t)|}{\lambda}\right) d t \leq 1\right\},
$$

and the pair $\left(L_{\mathscr{G}}([0,1]),\|\cdot\|_{\mathscr{G}}\right)$ is a Banach space.

For an Orlicz space, the Hölder inequality holds; that is,

$$
\int_{0}^{1} u v d t \leq 2\|u\|_{\mathscr{G}}\|v\|_{\mathscr{G}^{*}}
$$

where $u \in L_{\mathscr{G}}([0,1])$ and $v \in L_{\mathscr{G}^{*}}([0,1])$.

The existence and uniqueness of solutions of GBVP (1) rely on the following fixed point theorems [23].

Theorem 10. Let $\mathfrak{P}$ be a closed, convex, bounded, and nonempty subset of a Banach space $X$. Let $T_{1}, T_{2}$ be operators such that

(i) $T_{1}\left(u_{1}\right)+T_{2}\left(u_{2}\right)$ belong to $\mathfrak{P}$ whenever $u_{1}, u_{2} \in \mathfrak{P}$

(ii) $T_{1}$ is a compact and $T_{2}$ is a contraction mapping

Then, there exists $u_{0} \in \mathfrak{P}$ such that $u_{0}=T_{1}\left(u_{0}\right)+T_{2}\left(u_{0}\right)$.

Theorem 11. Let $X$ be a Banach space. Assume that $T: X$ $\longrightarrow X$ is a completely continuous operator and the set $V$ $=\{u \in X: u=\varepsilon T u, 0<\varepsilon<1\}$ is bounded. Then, $T$ has a fixed point in $X$.

For computational convenience,

$$
\begin{aligned}
\phi(t) & =\frac{1}{\psi_{1}}\left[\frac{\Gamma(\alpha) t^{\rho(\alpha+\beta-1)}}{\rho^{\beta} \Gamma(\alpha+\beta)}+\frac{\psi_{3}}{\psi_{2}}\right] \\
\psi_{1} & =\frac{\Gamma(\alpha)}{\rho^{\beta-1} \Gamma(\alpha+\beta-1)}\left[1-b_{1} \sum_{i=1}^{m} \mu_{i} \frac{\xi_{i}^{\rho(\alpha+\beta+q-2)} \Gamma(\alpha+\beta-1)}{\rho^{q} \Gamma(\alpha+\beta+q-1)}-b_{2} \sum_{i=1}^{p} r_{i} \eta_{i}^{\rho(\alpha+\beta-2)}\right] \neq 0 \\
\psi_{2} & =1-a_{1} \sum_{i=1}^{m} \sigma_{i} \frac{\xi_{i}^{\rho q}}{\rho^{q} \Gamma(q+1)}-a_{2} \sum_{i=1}^{p} \delta_{i} \neq 0 \\
\psi_{3} & =a_{1} \sum_{i=1}^{m} \sigma i \frac{\Gamma(\alpha) \xi_{i}^{\rho(\alpha+\beta+q-1)}}{\rho^{\beta+q} \Gamma(\alpha+\beta+q)}+a_{2} \sum_{i=1}^{p} \delta_{i} \frac{\Gamma(\alpha) \eta_{i}^{\rho(\alpha+\beta-1)}}{\rho^{\beta} \Gamma(\alpha+\beta)} .
\end{aligned}
$$


Lemma 12. Let $h \in L_{\mathscr{G}}([0,1])$. Then, the solution of the following GBVP:

$$
\left\{\begin{array}{l}
{ }^{R L} D_{0^{+}}^{\alpha, \rho}\left({ }^{C} D_{0^{+}}^{\beta, \rho} y(t)\right)=h(t), \quad 0<\alpha, \beta \leq 1, \\
y(0)=\alpha_{1} \sum_{i=1}^{m} \sigma_{i} I_{0^{+}}^{q, \rho} y\left(\varepsilon_{i}\right)+\alpha_{2} \sum_{i=1}^{p} \delta_{i} y\left(\eta_{i}\right), \\
\gamma y(1)=b_{1} \sum_{i=1}^{m} \mu_{i} I_{0^{+}}^{q, \rho} \gamma y\left(\varepsilon_{i}\right)+b_{2} \sum_{i=1}^{p} r_{i} \gamma y\left(\eta_{i}\right),
\end{array}\right.
$$

is given by

$$
\begin{aligned}
y(t)= & I_{0^{+}}^{\alpha+\beta, \rho} h(t)+\phi(t)\left[b_{1} \sum_{i=1}^{m} \mu_{i} I_{0^{+}}^{\alpha+\beta+q-1, \rho} h\left(\xi_{i}\right)\right. \\
& \left.+b_{2} \sum_{i=1}^{p} r_{i} I_{0^{+}}^{\alpha+\beta-1, \rho} h\left(\eta_{i}\right)-I_{0^{+}}^{\alpha+\beta-1, \rho} h(1)\right] \\
& +\frac{1}{\psi_{2}}\left[a_{1} \sum_{i=1}^{m} \sigma_{i} I_{0^{+}}^{\alpha+\beta+q, \rho} h\left(\xi_{i}\right)+a_{2} \sum_{i=1}^{p} \delta_{i} I_{0^{+}}^{\alpha+\beta, \rho} h\left(\eta_{i}\right)\right],
\end{aligned}
$$

where $\phi$ and $\psi_{2}$ are given in (23).

Proof. To obtain the integral equation modeled by the GBVP(24), we apply the generalization Riemann-Liouville fractional integral of order $\alpha$ to both sides of (24), and we get

$$
{ }^{\mathrm{C}} D_{0^{+}}^{\beta, \rho}(y(t))=I_{0^{+}}^{\alpha, \rho} h(t)+c_{1} t^{\rho(\alpha-1)} .
$$

Next, applying the generalization Caputo fractional integral of order $\beta$ to both sides of (26), we have

$$
y(t)=I_{0^{+}}^{\alpha+\beta, \rho} h(t)+c_{1} I_{0^{+}}^{\beta, \rho} t^{\rho(\alpha-1)}+c_{2},
$$

where $c_{1}$ and $c_{2} \in \mathbb{R}$. By using Lemma 7 , we get

$$
y(t)=I_{0^{+}}^{\alpha+\beta, \rho} h(t)+c_{1} \frac{t^{\rho(\alpha+\beta-1)} \Gamma(\alpha)}{\rho^{\beta} \Gamma(\alpha+\beta)}+c_{2} .
$$

So, the quantities $\gamma y(t)$ and $I^{q, \rho} \gamma y(t)$ are given, respectively, by

$$
\begin{aligned}
\gamma y(t) & =I^{\alpha+\beta-1, \rho} h(t)+c_{1} \frac{t^{\rho(\alpha+\beta-2)} \Gamma(\alpha)}{\rho^{\beta} \Gamma(\alpha+\beta-1)}, \\
I^{q, \rho} \gamma y(t) & =I^{\alpha+\beta+q-1, \rho} h(t)+\frac{c_{1} \Gamma(\alpha)}{\rho^{\beta} \Gamma(\alpha+\beta-1)} \frac{t^{\rho(\alpha+\beta+q-2)} \Gamma(\alpha+\beta)}{\rho^{q} \Gamma(\alpha+\beta+q)} .
\end{aligned}
$$

In view of the boundary conditions given in (24), the con- stants $c_{1}$ and $c_{2}$ are defined by

$$
\begin{aligned}
c_{2}= & \frac{1}{\psi_{2}}\left[\frac { \psi _ { 3 } } { \psi _ { 1 } } \left(b_{1} \sum_{i=1}^{m} \mu_{i} I_{0^{+}}^{\alpha+\beta+q-1, \rho} h\left(\xi_{i}\right)+b_{2} \sum_{i=1}^{p} r_{i} I_{0^{+}}^{\alpha+\beta-1, \rho} h\left(\eta_{i}\right)\right.\right. \\
& \left.\left.-I_{0^{+}}^{\alpha+\beta-1, \rho} h(1)+a_{1} \sum_{i=1}^{m} \sigma_{i} I_{0^{+}}^{\alpha+\beta+q, \rho} h\left(\xi_{i}\right)+a_{2} \sum_{i=1}^{p} \delta_{i} I_{0^{+}}^{\alpha+\beta, \rho} h\left(\eta_{i}\right)\right)\right], \\
c_{1}= & \frac{1}{\psi_{1}}\left[b_{1} \sum_{i=1}^{m} \mu_{i} I_{0^{+}}^{\alpha+\beta+q-1, \rho} h\left(\xi_{i}\right)+b_{2} \sum_{i=1}^{p} r_{i} I_{0^{+}}^{\alpha+\beta-1, \rho} h\left(\eta_{i}\right)-I_{0^{+}}^{\alpha+\beta-1, \rho} h(1)\right],
\end{aligned}
$$

where $\psi_{1}, \psi_{2}, \psi_{3}$, and $\phi(t)$ are given in (23), and by substituting of values of $c_{1}$ and $c_{2}$, we obtain (25).

\section{Main Results}

In this section, we are looking to prove the existence and uniqueness of solutions of GBVP (1). We will utilize in our proofs the fixed point theorems. We shall assume that $g$ is in the Orlicz space $L_{\mathscr{G}}[0,1]$. Let $Y=\{y: y \in C([0,1], \mathbb{R})\}$ denote the Banach space of all continuous functions on $[0,1]$ into $\mathbb{R}$ endowed with the norm $\|y\|=\sup \{|y(t)|: t$ $\epsilon[0,1]\}$. Now, we define an operator $\mathscr{F}: Y \longrightarrow Y$ associated with problem (1) by

$$
\begin{aligned}
(\mathscr{F} y)(t)= & I_{0^{+}}^{\alpha+\beta, \rho} g(t, y(t))+\phi(t)\left[b_{1} \sum_{i=1}^{m} \mu_{i} I_{0^{+}}^{\alpha+\beta+q-1, \rho} g\left(\xi_{i}, y\left(\xi_{i}\right)\right)\right. \\
& \left.+b_{2} \sum_{i=1}^{p} r_{i} I_{0^{+}}^{\alpha+\beta-1, \rho} g\left(\eta_{i}, y\left(\eta_{i}\right)\right)-I_{0^{+}}^{\alpha+\beta-1, \rho} g(1, y(1))\right] \\
& +\frac{1}{\psi_{2}}\left[a_{1} \sum_{i=1}^{m} \sigma_{i} I_{0^{+}}^{\alpha+\beta+q, \rho} g\left(\xi_{i}, g\left(\xi_{i}\right)\right)\right. \\
& \left.+a_{2} \sum_{i=1}^{p} \delta_{i} I_{0^{+}}^{\alpha+\beta, \rho} g\left(\eta_{i}, y\left(\eta_{i}\right)\right)\right] .
\end{aligned}
$$

Therefore, the GBVP (1) has a solution if and only if the operator $\mathscr{F}$ has a fixed point.

The following lemma plays a curial role in the sequel.

Lemma 13. Let $\alpha, \beta \in(0,1], q \in(0,1)$.Let $\mathscr{G}$ be a Young function which has a Young complement function $\mathscr{G}^{*}$ satisfying

$$
\int_{0}^{t} \mathscr{G}^{*}\left(s^{\gamma}\right) d s<\infty
$$

where $\gamma \in\{\alpha+\beta, \alpha+\beta-1, \alpha+\beta+q, \alpha+\beta+q-1\}$. Then, the operator $\mathscr{F}$ exists and is well defined.

Proof. Let $\alpha, \beta \in(0,1], q \in(0,1)$, and $y \in Y$. Define a function

$$
\Lambda_{1}(s)=\left\{\begin{array}{l}
\left(t^{\rho}-s^{\rho}\right)^{\alpha+\beta} s^{\rho-1} \\
0, \quad \text { otherwise, }
\end{array}\right.
$$


We show that $\Lambda_{1} \in L_{\mathscr{G}^{*}}[0,1]$. By using appropriate substitution and properties of the Young functions, one obtains

$$
\begin{aligned}
\int_{0}^{1} \mathscr{G}^{*}\left(\frac{\left|\Lambda_{1}(s)\right|}{k}\right) d s & =\int_{0}^{t} \mathscr{G}^{*}\left(\frac{\left(t^{\rho}-s^{\rho}\right)^{\alpha+\beta}}{k}\right) s^{\rho-1} d s \\
& =\frac{1}{\rho} k^{1 /(\alpha+\beta)} \int_{0}^{\left(1 / k^{(1 /(\alpha+\beta) \alpha+\beta) 1 / \alpha+\beta}\right)^{t^{\rho}}} \mathscr{G}^{*}\left(s^{\alpha+\beta}\right) d s .
\end{aligned}
$$

By the assumption of the theorem, we get $\Lambda_{1} \in L_{\mathscr{G}^{*}}[0,1]$. Similarly, set

$$
\begin{aligned}
& \Lambda_{2}(s)=\left\{\begin{array}{l}
\left.t^{\rho}-s^{\rho}\right)^{\alpha+\beta-1} s^{\rho-1} \text { if } \in[0, t], t>0, \\
0, \text { otherwise, }
\end{array}\right. \\
& \Lambda_{3}(s)=\left\{\begin{array}{l}
\left(t^{\rho}-s^{\rho}\right)^{\alpha+\beta+q} s^{\rho-1} \text { if } s \in[0, t], t>0, \\
0, \text { otherwise, }
\end{array}\right. \\
& \Lambda_{4}(s)= \begin{cases}\left(t^{\rho}-s^{\rho}\right)^{\alpha+\beta+q-1} s^{\rho-1} & \text { if } s \in[0, t], t>0, \\
0, & \text { otherwise. }\end{cases}
\end{aligned}
$$

Then, we can get $\Lambda_{2}, \Lambda_{3}$, and $\Lambda_{4} \in L_{\mathscr{G}^{*}}[0,1]$. Next, we show that $\mathscr{F}$ is well defined, i.e., $\mathscr{F} y(t) \in C([0,1], \mathbb{R})$. Let 0 $\leq \tau \leq t \leq 1$, then we have

$$
\begin{aligned}
|(\mathscr{F} y)(t)-(\mathscr{F} y)(\tau)| \leq & \sup _{t \in[0,1]}\left\{\frac{1}{\rho^{\alpha+\beta-1} \Gamma(\alpha+\beta)} \int_{0}^{\tau}\right. \\
& \left.\cdot\left(\mid\left(t^{\rho}-s^{\rho}\right)^{\alpha+\beta-1}-\left(\tau^{\rho}-s^{\rho}\right)^{\alpha+\beta-1}\right) s^{\rho-1} \mid\right) \\
& \cdot|g(s, y(s))| d s+\frac{1}{\rho^{\alpha+\beta-1} \Gamma(\alpha+\beta)} \\
& \cdot \int_{\tau}^{t}\left|\left(t^{\rho}-s^{\rho}\right)^{\alpha+\beta-1} s^{\rho-1}\right| g(s, y(s)) \mid d s \\
& +\frac{1}{\psi_{1}}\left[\frac{\Gamma(\alpha)\left|t^{\rho(\alpha+\beta-1)}-\tau^{\rho(\alpha+\beta-1)}\right|}{\rho^{\beta} \Gamma(\alpha+\beta)}\right] \\
& \cdot\left[b_{1} \sum_{i=1}^{m} \mu_{i} \frac{1}{\rho^{\alpha+\beta+q-2} \Gamma(\alpha+\beta+q-1)}\right. \\
& \cdot \int_{0}^{\xi_{i}}\left(\xi_{i}^{\rho}-s^{\rho}\right)^{\alpha+\beta+q-2} s^{\rho-1}|g(s, y(s))| d s \\
& +b_{2} \sum_{i=1}^{p} r_{i} \frac{1}{\rho^{\alpha+\beta-2} \Gamma(\alpha+\beta-1)} \\
& \cdot \int_{0}^{\eta_{i}}\left(\eta_{i}^{\rho}-s^{\rho}\right)^{\alpha+\beta-2} s^{\rho-1}|g(s, y(s))| d s \\
& -\frac{1}{\rho^{\alpha+\beta-2} \Gamma(\alpha+\beta-1)} \\
& \left.\cdot \int_{0}^{1}\left(1-s^{\rho}\right)^{\alpha+\beta-2} s^{\rho-1}|g(s, y(s))| d s\right]
\end{aligned}
$$

$$
\begin{aligned}
& +\frac{1}{\left|\psi_{2}\right|}\left[a_{1} \sum_{i=1}^{m} \sigma_{i} \frac{1}{\rho^{\alpha+\beta+q-1} \Gamma(\alpha+\beta+q)}\right. \\
& \cdot \int_{0}^{\xi_{i}}\left(\xi_{i}^{\rho}-s^{\rho}\right)^{\alpha+\beta+q-1} s^{\rho-1}|g(s, y(s))| d s \\
& +a_{2} \sum_{i=1}^{p} \delta_{i} \frac{1}{\rho^{\alpha+\beta-1} \Gamma(\alpha+\beta)} \\
& \left.\left.\cdot \int_{0}^{\eta_{i}}\left(\eta_{i}^{\rho}-s^{\rho}\right)^{\alpha+\beta-1} s^{\rho-1}|g(s, y(s))| d s\right]\right\} \\
& =\frac{1}{\rho^{\alpha+\beta-1} \Gamma(\alpha+\beta)} \int_{0}^{1}\left[\chi_{1}(s)+\chi_{2}(s)\right]|g(s, y(s))| d s \\
& +\frac{1}{\psi_{1}}\left[\frac{\Gamma(\alpha)\left|t^{\rho(\alpha+\beta-1)}-\tau^{\rho(\alpha+\beta-1)}\right|}{\rho^{\beta} \Gamma(\alpha+\beta)}\right] \\
& \cdot\left[b_{1} \sum_{i=1}^{m} \mu_{i} \frac{1}{\rho^{\alpha+\beta+q-2} \Gamma(\alpha+\beta+q-1)}\right. \\
& \cdot \int_{0}^{\xi_{i}}\left(\xi_{i}^{\rho}-s^{\rho}\right)^{\alpha+\beta+q-2} s^{\rho-1}|g(s, y(s))| d s \\
& +b_{2} \sum_{i=1}^{p} r_{i} \frac{1}{\rho^{\alpha+\beta-2} \Gamma(\alpha+\beta-1)} \\
& \cdot \int_{0}^{\eta_{i}}\left(\eta_{i}^{\rho}-s^{\rho}\right)^{\alpha+\beta-2} s^{\rho-1}|g(s, y(s))| d s \\
& \left.-\frac{1}{\rho^{\alpha+\beta-2} \Gamma(\alpha+\beta-1)} \int_{0}^{1}\left(1-s^{\rho}\right)^{\alpha+\beta-2} s^{\rho-1}|g(s, y(s))| d s\right] \\
& +\frac{1}{\left|\psi_{2}\right|}\left[a_{1} \sum_{i=1}^{m} \sigma_{i} \frac{1}{\rho^{\alpha+\beta+q-1} \Gamma(\alpha+\beta+q)}\right. \\
& \cdot \int_{0}^{\xi_{i}}\left(\xi_{i}^{\rho}-s^{\rho}\right)^{\alpha+\beta+q-1} s^{\rho-1}|g(s, y(s))| d s \\
& +a_{2} \sum_{i=1}^{p} \delta_{i} \frac{1}{\rho^{\alpha+\beta-1} \Gamma(\alpha+\beta)} \\
& \left.\left.\cdot \int_{0}^{\eta_{i}}\left(\eta_{i}^{\rho}-s^{\rho}\right)^{\alpha+\beta-1} s^{\rho-1}|g(s, y(s))| d s\right]\right\} \text {, }
\end{aligned}
$$

where

$$
\begin{aligned}
& \chi_{1}(s)=\left\{\begin{array}{l}
\left|\left(\left(t^{\rho}-s^{\rho}\right)^{\alpha+\beta-1}-\left(\tau^{\rho}-s^{\rho}\right)^{\alpha+\beta-1}\right) s^{\rho-1}\right| \quad \text { if } s \in[0, \tau], \\
0, \quad \text { otherwise, }
\end{array}\right. \\
& \chi_{2}(s)= \begin{cases}\left|\left(t^{\rho}-s^{\rho}\right)^{\alpha+\beta-1} s^{\rho-1}\right| & \text { if } s \in[0, \tau], \\
0, & \text { otherwise. }\end{cases}
\end{aligned}
$$

The functions $\chi_{i}, i=1,2$ belong to $L_{\mathscr{G}^{*}}[0,1]$ with $\left\|\chi_{i}\right\|_{\mathscr{G}^{*}}$ $\leq J\left(\left|t^{\rho}-\tau^{\rho}\right|\right), i=1,2$, where $J: \mathbb{R}^{+} \longrightarrow \mathbb{R}^{+}$is a continuous, 
increasing function with $J(0)=0$. Using the Hölder inequality, we get

$$
\begin{aligned}
& |(\mathscr{F} y)(t)-(\mathscr{F} y)(\tau)| \leq\|g\|_{\mathscr{G}^{*}}\left(\frac{1}{2 \rho^{\alpha+\beta-1} \Gamma(\alpha+\beta)}\left[\left\|\chi_{1}\right\|_{\mathscr{G}^{*}}+\left\|\chi_{2}\right\|_{\mathscr{G}^{*}}\right]\right. \\
& +\frac{1}{\psi_{1}}\left[\frac{\Gamma(\alpha)\left|t^{\rho(\alpha+\beta-1)}-\tau^{\rho(\alpha+\beta-1)}\right|}{\rho^{\beta} \Gamma(\alpha+\beta)}\right] \\
& \quad \cdot\left[b_{1} \sum_{i=1}^{m} \mu_{i} \frac{\left(\xi_{i}\right)^{\rho(\alpha+\beta+q-1)}}{\rho^{\alpha+\beta+q-1} \Gamma(\alpha+\beta+q)}\right. \\
& \left.+\frac{1}{\rho^{\alpha+\beta-1} \Gamma(\alpha+\beta)}\left(b_{2} \sum_{i=1}^{p} r_{i}\left(\eta_{i}\right)^{\rho(\alpha+\beta-1)}-1\right)\right] \\
& +\frac{1}{\left|\psi_{2}\right|}\left[a_{1} \sum_{i=1}^{m} \sigma_{i} \frac{\left(\xi_{i}\right)^{\rho(\alpha+\beta+q)}}{\rho^{\alpha+\beta+q} \Gamma(\alpha+\beta+q+1)}\right. \\
& \left.\left.+a_{2} \sum_{i=1}^{p} \delta_{i} \frac{\left(\eta_{i}\right)^{\rho(\alpha+\beta)}}{\rho^{\alpha+\beta} \Gamma(\alpha+\beta+1)}\right]\right) .
\end{aligned}
$$

Hence, for $0<\left|t^{\rho}-\tau^{\rho}\right|<\varepsilon$ and by continuity of $J$, we see that $\mathscr{F}$ is continuous, which completes the proof.

For convenience, we define

$$
\begin{aligned}
\psi= & \frac{1}{\rho^{\alpha+\beta} \Gamma(\alpha+\beta+1)}+\|\phi\|\left[b_{1} \sum_{i=1}^{m} \mu_{i} \frac{\left(\xi_{i}\right)^{\rho(\alpha+\beta+q-1)}}{\rho^{\alpha+\beta+q-1} \Gamma(\alpha+\beta+q)}\right. \\
& \left.+\frac{1}{\rho^{\alpha+\beta-1} \Gamma(\alpha+\beta)}\left[b_{2} \sum_{i=1}^{p} r_{i}\left(\eta_{i}\right)^{\rho(\alpha+\beta-1)}-1\right]\right] \\
& +\frac{1}{\left|\psi_{2}\right|}\left[a_{1} \sum_{i=1}^{m} \sigma_{i} \frac{\left(\xi_{i}\right)^{\rho(\alpha+\beta+q)}}{\rho^{\alpha+\beta+q} \Gamma(\alpha+\beta+q+1)}\right. \\
& \left.+a_{2} \sum_{i=1}^{p} \delta_{i} \frac{\left(\eta_{i}\right)^{\rho(\alpha+\beta)}}{\rho^{\alpha+\beta} \Gamma(\alpha+\beta+1)}\right] .
\end{aligned}
$$

Now, we are in a position to state and prove the first existence result. The following result is based on Banach fixed point theorem.

Theorem 14. Assume that there exists a constant $\Omega>0$ such that

(S1) $|g(t, x)-g(t, y)| \leq \Omega|x-y| \quad$ for all $t \in[0,1]$ and $x, y \in \mathbb{R}$.

If $\Omega \psi<1$ where $\psi$ is given by (39), then the boundary value problem (1) has a unique solution on $[0,1]$.

Proof. We define $B_{r}=\{y \in Y:\|y\| \leq r\}$, with $r \geq g^{*} \psi /(1-\Omega$ $\psi)$, and $\psi$ is defined by (39). Set

$$
g^{*}=\sup _{t \in[0,1]}|g(t, 0)|<\infty
$$

We shall show that $\mathscr{F} B_{r} \subset B_{r}$. For any $y \in B_{r}$, by assumption (S1), we obtain that
$\|\mathscr{F} y\| \leq\left(\Omega\|y\|+g^{*}\right)\left\{\frac{1}{\rho^{\alpha+\beta-1} \Gamma(\alpha+\beta)} \int_{0}^{t}\left(t^{\rho}-s^{\rho}\right)^{\alpha+\beta-1} s^{\rho-1} d s\right.$

$$
+\|\phi\|\left[b_{1} \sum_{i=1}^{m} \mu_{i} \frac{1}{\rho^{\alpha+\beta+q-2} \Gamma(\alpha+\beta+q-1)}\right.
$$$$
\cdot \int_{0}^{\xi_{i}}\left(\xi_{i}^{\rho}-s^{\rho}\right)^{\alpha+\beta+q-2} s^{\rho-1} d s+b_{2} \sum_{i=1}^{p} r_{i} \frac{1}{\rho^{\alpha+\beta-2} \Gamma(\alpha+\beta-1)}
$$$$
\cdot \int_{0}^{\eta_{i}}\left(\eta_{i}^{\rho}-s^{\rho}\right)^{\alpha+\beta-2} s^{\rho-1} d s-\frac{1}{\rho^{\alpha+\beta-2} \Gamma(\alpha+\beta-1)}
$$$$
\left.\int_{0}^{1}\left(1-s^{\rho}\right)^{\alpha+\beta-2} s^{\rho-1} d s\right]
$$$$
+\frac{1}{\left|\psi_{2}\right|}\left[a_{1} \sum_{i=1}^{m} \sigma_{i} \frac{1}{\rho^{\alpha+\beta+q-1} \Gamma(\alpha+\beta+q)}\right.
$$$$
\cdot \int_{0}^{\xi_{i}}\left(\xi_{i}^{\rho}-s^{p}\right)^{\alpha+\beta+q-1} s^{\rho-1} d s+a_{2} \sum_{i=1}^{p} \delta_{i} \frac{1}{\rho^{\alpha+\beta-1} \Gamma(\alpha+\beta)}
$$$$
\left.\left.\int_{0}^{\eta_{i}}\left(\eta_{i}^{\rho}-s^{\rho}\right)^{\alpha+\beta-1} s^{\rho-1} d s\right]\right\}
$$$$
\leq\left(\Omega r+g^{*}\right)\left\{\frac{1}{\rho^{\alpha+\beta} \Gamma(\alpha+\beta+1)}\right.
$$$$
+\|\phi\|\left[b_{1} \sum_{i=1}^{m} \mu_{i} \frac{\left(\xi_{\mathrm{i}}\right)^{\rho(\alpha+\beta+q-1)}}{\rho^{\alpha+\beta+q-1} \Gamma(\alpha+\beta+q)}+\frac{1}{\rho^{\alpha+\beta-1} \Gamma(\alpha+\beta)}\right.
$$$$
\left.\cdot\left[b_{2} \sum_{i=1}^{p} r_{i}\left(\eta_{i}\right)^{\rho(\alpha+\beta-1)}-1\right]\right]
$$$$
+\frac{1}{\left|\psi_{2}\right|}\left[a_{1} \sum_{i=1}^{m} \sigma_{i} \frac{\left(\xi_{i}\right)^{\rho(\alpha+\beta+q)}}{\rho^{\alpha+\beta+q} \Gamma(\alpha+\beta+q+1)}\right.
$$$$
\left.\left.+a_{2} \sum_{i=1}^{p} \delta_{i} \frac{\left(\eta_{i}\right)^{\rho(\alpha+\beta)}}{\rho^{\alpha+\beta} \Gamma(\alpha+\beta+1)}\right]\right\} \text {. }
$$

Therefore,

$$
\|\mathscr{F} y\| \leq\left(\Omega r+g^{*}\right) \psi \leq r .
$$

Now, for $y, \tilde{y} \in C([0,1], \mathbb{R})$, and for each $t \in[0,1]$, we get

$$
\begin{aligned}
\|\mathscr{F} y-\mathscr{F} y\| \leq & \sup _{t \in[0,1]}\left\{\frac{1}{\rho^{\alpha+\beta-1} \Gamma(\alpha+\beta)} \int_{0}^{t}\left(t^{\rho}-s^{\rho}\right)^{\alpha+\beta+1} s^{\rho-1} \mid g(s, y(s))\right. \\
& -g(s, \tilde{y}(s))|d s+| \phi(t) \mid \\
& \cdot\left[b_{1} \sum_{i=1}^{m} \mu_{i} \frac{1}{\rho^{\alpha+\beta+q-2} \Gamma(\alpha+\beta+q-1)}\right. \\
& \cdot \int_{0}^{\xi_{i}}\left(\xi_{i}^{\rho}-s^{\rho}\right)^{\alpha+\beta+q-2} s^{\rho-1}|g(s, y(s))-g(s, \tilde{y}(s))| d s \\
& +b_{2} \sum_{i=1}^{p} r_{i} \frac{1}{\rho^{\alpha+\beta-2} \Gamma(\alpha+\beta-1)} \\
& \cdot \int_{0}^{\eta_{i}}\left(\eta_{i}^{\rho}-s^{\rho}\right)^{\alpha+\beta-2} s^{\rho-1} \mid g(s, y(s))
\end{aligned}
$$




$$
\begin{aligned}
& -g(s, \tilde{y}(s)) \mid d s-\frac{1}{\rho^{\alpha+\beta-2} \Gamma(\alpha+\beta-1)} \\
& \left.\cdot \int_{0}^{1}\left(1-s^{\rho}\right)^{\alpha+\beta-2} s^{\rho-1}|g(s, y(s))-g(s, \tilde{y}(s)) s| d s\right] \\
& +\frac{1}{\left|\psi_{2}\right|}\left[a_{1} \sum_{i=1}^{m} \sigma_{i} \frac{1}{\rho^{\alpha+\beta+q-1} \Gamma(\alpha+\beta+q)}\right. \\
& \cdot \int_{0}^{\xi_{i}}\left(\xi_{i}^{\rho}-s^{\rho}\right)^{\alpha+\beta+q-1} s^{\rho-1}|g(s, y(s))-g(s, \tilde{y}(s))| d s \\
& +a_{2} \sum_{i=1}^{p} \delta_{i} \frac{1}{\rho^{\alpha+\beta-1} \Gamma(\alpha+\beta)} \\
& \left.\cdot \int_{0}^{\eta_{i}}\left(\eta_{i}^{\rho}-s^{\rho}\right)^{\alpha+\beta-1} s^{\rho-1}|g(s, y(s))-g(s, \tilde{y}(s))| d s\right\} \\
& \leq \Omega\|y-\tilde{y}\|\left[\frac{1}{\rho^{\alpha+\beta} \Gamma(\alpha+\beta+1)}+\|\phi\|\right. \\
& \cdot\left(b_{1} \sum_{i=1}^{m} \mu_{i} \frac{\left(\xi_{i}\right)^{\rho(\alpha+\beta+q-1)}}{\rho^{\alpha+\beta+q-1} \Gamma(\alpha+\beta+q)}+\frac{1}{\rho^{\alpha+\beta-1} \Gamma(\alpha+\beta)}\right. \\
& \left.\cdot\left[b_{2} \sum_{i=1}^{p} r_{i}\left(\eta_{i}\right)^{\rho(\alpha+\beta-1)}-1\right]\right)+\frac{1}{\left|\psi_{2}\right|} \\
& \cdot\left(a_{1} \sum_{i=1}^{m} \sigma_{i} \frac{\left(\xi_{i}\right)^{\rho(\alpha+\beta+q)}}{\rho^{\alpha+\beta+q} \Gamma(\alpha+\beta+q+1)}\right. \\
& \left.\left.+a_{2} \sum_{i=1}^{\rho} \delta_{i} \frac{\left(\eta_{i}\right)^{\rho(\alpha+\beta)}}{\rho^{\alpha+\beta} \Gamma(\alpha+\beta+1)}\right)\right] .
\end{aligned}
$$

So,

$$
\|(\mathscr{F} y)-(\mathscr{F} \tilde{y})\| \leq \psi \Omega\|y-\tilde{y}\| .
$$

As $\Omega \psi<1$, then $\mathscr{F}$ is a contraction. Hence, by the Banach fixed point theorem, $\mathscr{F}$ has a unique fixed point which is a unique solution of boundary value problem $(1)$ on $[0,1]$.

In the next result, we prove the existence of problem (1) by applying Schaefer's fixed point theorem.

\section{Theorem 15. Suppose that}

(S2) there exist a constant $v>0$ such that $|g(t, y)| \leq v$ for each $t \in[0,1]$ and all $y \in \mathbb{R}$.

Then, the boundary value problem (1) has at least one solution in $[0,1]$.

Proof. The proof is divided into three steps.

Step 1. Let $K$ be a bounded subset of $Y$. By (S2), for all $t \in[$ $0,1]$ and $y \in K$, we have

$$
\begin{gathered}
\|\mathscr{F} y\| \leq \sup _{t \in[0,1]}\left\{\frac{1}{\rho^{\alpha+\beta-1} \Gamma(\alpha+\beta)} \int_{0}^{t}\left(t^{\rho}-s^{\rho}\right)^{\alpha+\beta-1} s^{\rho-1}|g(s, y(s))| d s\right. \\
+|\phi(t)|\left[b_{1} \sum_{i=1}^{m} \mu_{i} \frac{1}{\rho^{\alpha+\beta+q-2} \Gamma(\alpha+\beta+q-1)}\right.
\end{gathered}
$$

$$
\begin{aligned}
& \cdot \int_{0}^{\xi_{i}}\left(\xi_{i}^{\rho}-s^{\rho}\right)^{\alpha+\beta+q-2} s^{\rho-1}|g(s, y(s))| d s \\
& +b_{2} \sum_{i=1}^{p} r_{i} \frac{1}{\rho^{\alpha+\beta-2} \Gamma(\alpha+\beta-1)} \\
& \cdot \int_{0}^{\eta_{i}}\left(\eta_{i}^{\rho}-s^{\rho}\right)^{\alpha+\beta-2} s^{\rho-1}|g(s, y(s))| d s \\
& \left.-\frac{1}{\rho^{\alpha+\beta-2} \Gamma(\alpha+\beta-1)} \int_{0}^{1}\left(1-s^{\rho}\right)^{\alpha+\beta-2} s^{\rho-1}|g(s, y(s))| d s\right] \\
& +\frac{1}{\left|\psi_{2}\right|}\left[a_{1} \sum_{i=1}^{m} \sigma_{i} \frac{1}{\rho^{\alpha+\beta+q-1} \Gamma(\alpha+\beta+q)}\right. \\
& \cdot \int_{0}^{\xi_{i}}\left(\xi_{i}^{\rho}-s^{\rho}\right)^{\alpha+\beta+q-1} s^{\rho-1}|g(s, y(s))| d s \\
& +a_{2} \sum_{i=1}^{p} \delta_{i} \frac{1}{\rho^{\alpha+\beta-1} \Gamma(\alpha+\beta)} \\
& \left.\left.\cdot \int_{0}^{\eta_{i}}\left(\eta_{i}^{\rho}-s^{\rho}\right)^{\alpha+\beta-1} s^{\rho-1}|g(s, y(s))| d s\right]\right\} \\
& \leq v\left\{\frac{1}{\rho^{\alpha+\beta} \Gamma(\alpha+\beta+1)}+\|\phi\|\right. \\
& \cdot\left[b_{1} \sum_{i=1}^{m} \mu_{i} \frac{\left(\xi_{i}\right)^{\rho(\alpha+\beta+q-1)}}{\rho^{\alpha+\beta+q-1} \Gamma(\alpha+\beta+q)}\right. \\
& \left.+\frac{1}{\rho^{\alpha+\beta-1} \Gamma(\alpha+\beta)}\left[b_{2} \sum_{i=1}^{p} r_{i}\left(\eta_{i}\right)^{\rho(\alpha+\beta-1)}-1\right]\right] \\
& +\frac{1}{\left|\psi_{2}\right|}\left[a_{1} \sum_{i=1}^{m} \sigma_{i} \frac{\left(\xi_{i}\right)^{\rho(\alpha+\beta+q)}}{\rho^{\alpha+\beta+q} \Gamma(\alpha+\beta+q+1)}\right. \\
& \left.\left.+a_{2} \sum_{i=1}^{p} \delta_{i} \frac{\left(\eta_{i}\right)^{\rho(\alpha+\beta)}}{\rho^{\alpha+\beta} \Gamma(\alpha+\beta+1)}\right]\right\} \\
& =v \psi=v^{*} \text {. }
\end{aligned}
$$

Then, $\|\mathscr{F} y\| \leq v^{*}$, and so $\mathscr{F}$ maps bounded sets into bounded sets in $Y$.

Step 2. Let $t_{1}, t_{2} \in[0,1], t_{1}<t_{2}$, and $K$ be a bounded set of $Y$. Thus, for $y \in K$ and by (S2), we have

$$
\begin{aligned}
\mid(\mathscr{F} y) & \left(t_{2}\right)-(\mathscr{F} y)\left(t_{1}\right) \mid \\
\leq & v\left\{\frac { 1 } { \rho ^ { \alpha + \beta - 1 } \Gamma ( \alpha + \beta ) } \left[\int_{0}^{t_{2}}\left|\left(t_{2}^{\rho}-s^{\rho}\right)^{\alpha+\beta-1} s^{\rho-1}\right| d s\right.\right. \\
& \left.-\int_{0}^{t_{1}}\left|\left(t_{1}^{\rho}-s^{\rho}\right)^{\alpha+\beta-1} s^{\rho-1}\right| d s\right] \\
& +\frac{1}{\psi_{1}}\left[\frac{\Gamma(\alpha)\left|t^{\rho(\alpha+\beta-1)}-\tau^{\rho(\alpha+\beta-1)}\right|}{\rho^{\beta} \Gamma(\alpha+\beta)}\right] \\
& \cdot\left[b_{1} \sum_{i=1}^{m} \mu_{i} \frac{1}{\rho^{\alpha+\beta+q-2} \Gamma(\alpha+\beta+q-1)}\right. \\
& \cdot \int_{0}^{\xi_{i}}\left|\left(\xi_{i}^{\rho}-s^{\rho}\right)^{\alpha+\beta+q-2} s^{\rho-1}\right| d s+b_{2} \sum_{i=1}^{p} r_{i} \frac{1}{\rho^{\alpha+\beta-2} \Gamma(\alpha+\beta-1)}
\end{aligned}
$$




$$
\begin{aligned}
& \cdot \int_{0}^{\eta_{i}}\left|\left(\eta_{i}^{\rho}-s^{\rho}\right)^{\alpha+\beta-2} s^{\rho-1}\right| d s-\frac{1}{\rho^{\alpha+\beta-2} \Gamma(\alpha+\beta-1)} \\
& \left.\cdot \int_{0}^{1}\left|\left(1-s^{\rho}\right)^{\alpha+\beta-2} s^{\rho-1}\right| d s\right]+\frac{1}{\left|\psi_{2}\right|} \\
& \cdot\left[a_{1} \sum_{i=1}^{m} \sigma_{i} \frac{1}{\rho^{\alpha+\beta+q-1} \Gamma(\alpha+\beta+q)}\right. \\
& \cdot \int_{0}^{\xi_{i}}\left|\left(\xi_{i}^{\rho}-s^{\rho}\right)^{\alpha+\beta+q-1} s^{\rho-1}\right| d s \\
& \left.\left.+a_{2} \sum_{i=1}^{p} \delta_{i} \frac{1}{\rho^{\alpha+\beta-1} \Gamma(\alpha+\beta)} \int_{0}^{\eta_{i}}\left|\left(\eta_{i}^{\rho}-s^{\rho}\right)^{\alpha+\beta-1} s^{\rho-1}\right| d s\right]\right\} \\
& \leq v\left\{\frac{1}{\rho^{\alpha+\beta} \Gamma(\alpha+\beta+1)}\left[2\left|t_{2}^{\rho}-t_{1}^{\rho}\right|^{\alpha+\beta}+\left|t_{2}^{\rho(\alpha+\beta)}-t_{1}^{\rho(\alpha+\beta)}\right|\right]\right. \\
& +\frac{1}{\psi_{1}}\left[\frac{\Gamma(\alpha)\left|t^{\rho(\alpha+\beta-1)}-\tau^{\rho(\alpha+\beta-1)}\right|}{\rho^{\beta} \Gamma(\alpha+\beta)}\right] \\
& \cdot\left[b_{1} \sum_{i=1}^{m} \mu_{i} \frac{\left(\xi_{i}\right)^{\rho(\alpha+\beta+q-1)}}{\rho^{\alpha+\beta+q-1} \Gamma(\alpha+\beta+q)}+\frac{1}{\rho^{\alpha+\beta-1} \Gamma(\alpha+\beta)}\right. \\
& \left.\cdot\left[b_{2} \sum_{i=1}^{p} r_{i}\left(\eta_{i}\right)^{\rho(\alpha+\beta-1)}-1\right]\right]+\frac{1}{\left|\psi_{2}\right|} \\
& \cdot\left[a_{1} \sum_{i=1}^{m} \sigma_{i} \frac{\left(\xi_{i}\right)^{\rho(\alpha+\beta+q)}}{\rho^{\alpha+\beta+q} \Gamma(\alpha+\beta+q+1)}\right. \\
& \left.\left.+a_{2} \sum_{i=1}^{p} \delta_{i} \frac{\left(\eta_{i}\right)^{\rho(\alpha+\beta)}}{\rho^{\alpha+\beta} \Gamma(\alpha+\beta+1)}\right]\right\} \text {. }
\end{aligned}
$$

It follows that the right-hand side tends to zero as $\left(t_{2}-\right.$ $\left.t_{1}\right) \longrightarrow 0$ independent of $y$; by Arzela-Ascoli theorem, we conclude that $\mathscr{F}$ is completely continuous.

Step 3. It remains to show that the set $M=\{y \in Y: y=a \mathscr{F}$ (y) for some $0<\mathrm{a}<1\}$ is a bounded set. Let $y \in M$, and then, $y(t)=a(\mathscr{F} y)(t), 0<\mathrm{a}<1, t \in[0,1]$. Hence, by (S2), we have

$$
\begin{aligned}
&\|y\| \leq \sup _{t \in[0,1]}\{ \frac{1}{\rho^{\alpha+\beta-1} \Gamma(\alpha+\beta)} \int_{0}^{t}\left(t^{\rho}-s^{\rho}\right)^{\alpha+\beta-1} s^{\rho-1}|g(s, y(s))| d s \\
&+|\phi(t)|\left[b_{1} \sum_{i=1}^{m} \mu_{i} \frac{1}{\rho^{\alpha+\beta+q-2} \Gamma(\alpha+\beta+q-1)}\right. \\
& \cdot \int_{0}^{\xi_{i}}\left(\xi_{i}^{\rho}-s^{\rho}\right)^{\alpha+\beta+q-2} s^{\rho-1}|g(s, y(s))| d s \\
&+ b_{2} \sum_{i=1}^{p} r_{i} \frac{1}{\rho^{\alpha+\beta-2} \Gamma(\alpha+\beta-1)} \\
& \cdot \int_{0}^{\eta_{i}}\left(\eta_{i}^{\rho}-s^{\rho}\right)^{\alpha+\beta-2} s^{\rho-1}|g(s, y(s))| d s \\
&\left.-\frac{1}{\rho^{\alpha+\beta-2} \Gamma(\alpha+\beta-1)} \int_{0}^{1}\left(1-s^{\rho}\right)^{\alpha+\beta-2} s^{\rho-1}|g(s, y(s))| d s\right]
\end{aligned}
$$

$$
\begin{gathered}
+\frac{1}{\left|\psi_{2}\right|}\left[a_{1} \sum_{i=1}^{m} \sigma_{i} \frac{1}{\rho^{\alpha+\beta+q-1} \Gamma(\alpha+\beta+q)}\right. \\
\quad \cdot \int_{0}^{\xi_{i}}\left(\xi_{i}^{\rho}-s^{\rho}\right)^{\alpha+\beta+q-1} s^{\rho-1}|g(s, y(s))| d s \\
+a_{2} \sum_{i=1}^{p} \delta_{i} \frac{1}{\rho^{\alpha+\beta-1} \Gamma(\alpha+\beta)} \\
\left.\left.\quad \cdot \int_{0}^{\eta_{i}}\left(\eta_{i}^{\rho}-s^{\rho}\right)^{\alpha+\beta-1} s^{\rho-1}|g(s, y(s))| d s\right]\right\} \\
\leq v\left[\frac{1}{\rho^{\alpha+\beta} \Gamma(\alpha+\beta+1)}+\|\phi\|\right. \\
\cdot\left(b_{1} \sum_{i=1}^{m} \mu_{i} \frac{\left(\xi_{i}\right)^{\rho(\alpha+\beta+q-1)}}{\rho^{\alpha+\beta+q-1} \Gamma(\alpha+\beta+q)}+\frac{1}{\rho^{\alpha+\beta-1} \Gamma(\alpha+\beta)}\right. \\
\left.\quad\left[b_{2} \sum_{i=1}^{p} r_{i}\left(\eta_{i}\right)^{\rho(\alpha+\beta-1)}-1\right]\right) \\
+\frac{1}{\left|\psi_{2}\right|}\left(a_{1} \sum_{i=1}^{m} \sigma_{i} \frac{\left(\xi_{i}\right)^{\rho(\alpha+\beta+q)}}{\rho^{\alpha+\beta+q} \Gamma(\alpha+\beta+q+1)}\right. \\
\left.\left.+a_{2} \sum_{i=1}^{p} \delta_{i} \frac{\left(\eta_{i}\right)^{\rho(\alpha+\beta)}}{\rho^{\alpha+\beta} \Gamma(\alpha+\beta+1)}\right)\right] \\
\leq v \psi \\
.
\end{gathered}
$$

This shows that $M$ is bounded. Schaefer's fixed point theorem guarantees the existence of a fixed point of $\mathscr{F}$. Hence, the integral equation (31) has a solution on $[0,1]$.

Now, we establish the existence of solutions of problem (1) via Krasnoselskii's fixed point theorem.

Theorem 16. Assume that $g:[0,1] \times \mathbb{R} \longrightarrow \mathbb{R}$ is a continuous function such that it satisfies (S1). In addition, we suppose that

(S3) $|g(t, x)| \leq \zeta(t)$ for $t \in[0,1]$ and $\zeta \in C([0,1], \mathbb{R})$ with $\|$ $\zeta \|=\max _{t \in[0,1]}|\zeta(t)|$.

Then, the boundary value problem (1) has at least one solution on $[0,1]$, provided that

$$
\Omega \psi^{*}<1
$$

where $\psi^{*}=\psi-1 /\left(\rho^{(\alpha+\beta)} \Gamma(\alpha+\beta+1)\right)$.

Proof. We define

$$
\sup _{t \in[0,1]}|\zeta(t)|=\|\zeta\|
$$

and choose a suitable constant $r^{*}$ such that $r^{*} \geq\|\zeta\| \psi$, where $\psi$ is given by (39). Furthermore, we split $\mathscr{F}$ into two 
operators $\mathscr{F}_{1}$ and $\mathscr{F}_{2}$ on $B_{r^{*}}=\left\{y \in Y:\|y\| \leq r^{*}\right\}$ by

$$
\begin{aligned}
& \mathscr{F}_{1} y=I_{0^{+}}^{\alpha+\beta, \rho} g(t, y(t)), \quad \text { for all } t \in[0,1], \\
& \mathscr{F}_{2} y= \phi(t)\left[b_{1} \sum_{i=1}^{m} \mu_{i} I_{0^{+}}^{\alpha+\beta+q-1, \rho} g\left(\xi_{i}, y\left(\xi_{i}\right)\right)\right. \\
&\left.+b_{2} \sum_{i=1}^{p} r_{i} I_{0^{+}}^{\alpha+\beta-1, \rho} g\left(\eta_{i}, y\left(\eta_{i}\right)\right)-I_{0^{+}}^{\alpha+\beta-1, \rho} g(1, y(1))\right] \\
&+\frac{1}{\psi_{2}}\left[a_{1} \sum_{i=1}^{m} \sigma_{i} I_{0^{+}}^{\alpha+\beta+q, \rho} g\left(\xi_{i}, y\left(\xi_{i}\right)\right)\right. \\
&\left.+a_{2} \sum_{i=1}^{p} \delta_{i} I_{0^{+}}^{\alpha+\beta, \rho} g\left(\eta_{i}, y\left(\eta_{i}\right)\right)\right] .
\end{aligned}
$$

For $y \in B_{r^{*}}$, we get

$$
\begin{aligned}
\left\|\mathscr{F}_{1} y+\mathscr{F}_{2} y\right\| \leq & \sup _{t \in[0,1]}\left\{\frac{1}{\rho^{\alpha+\beta-1} \Gamma(\alpha+\beta)}\right. \\
& \cdot \int_{0}^{t}\left(t^{\rho}-s^{\rho}\right)^{\alpha+\beta-1} s^{\rho-1}|g(s, y(s))| d s \\
& +|\phi(t)|\left[b_{1} \sum_{i=1}^{m} \mu_{i} \frac{1}{\rho^{\alpha+\beta+q-2} \Gamma(\alpha+\beta+q-1)}\right. \\
& \cdot \int_{0}^{\xi_{i}}\left(\xi_{i}^{\rho}-s^{\rho}\right)^{\alpha+\beta+q-2} s^{\rho-1}|g(s, y(s))| d s \\
& +b_{2} \sum_{i=1}^{p} r_{i} \frac{1}{\rho^{\alpha+\beta-2} \Gamma(\alpha+\beta-1)} \\
& \cdot \int_{0}^{\eta_{i}}\left(\eta_{i}^{\rho}-s^{\rho}\right)^{\alpha+\beta-2} s^{\rho-1}|g(s, y(s))| d s \\
& -\frac{1}{\rho^{\alpha+\beta-2} \Gamma(\alpha+\beta-1)} \\
& \left.\cdot \int_{0}^{1}\left(1-s^{\rho}\right)^{\alpha+\beta-2} s^{\rho-1}|g(s, y(s))| d s\right] \\
& +\frac{1}{\left|\psi_{2}\right|}\left[a_{1} \sum_{i=1}^{m} \sigma_{i} \frac{1}{\rho^{\alpha+\beta+q-1} \Gamma(\alpha+\beta+q)}\right. \\
& \cdot \int_{0}^{\xi_{i}}\left(\xi_{i}^{\rho}-s^{\rho}\right)^{\alpha+\beta+q-1} s^{\rho-1}|g(s, y(s))| d s \\
& \left.\left.+a_{2} \sum_{i=1}^{p} \delta_{i} \frac{1}{\rho^{\alpha+\beta-1} \Gamma(\alpha+\beta)}\left(\eta_{i}^{\rho}-s^{\rho}\right)^{\alpha+\beta-1} s^{\rho-1}|g(s, y(s))| d s\right]\right\} \\
& \eta_{i}^{*},
\end{aligned}
$$

which shows that $\mathscr{F}_{1} y+\mathscr{F}_{2} y \in B_{r^{*}}$. Now, we need to prove that $\mathscr{F}_{2}$ is a contraction. By assumption (S1), we have

$$
\begin{aligned}
\left\|\mathscr{F}_{2} y-\mathscr{F}_{2} \tilde{y}\right\| \leq & \sup _{t \in[0,1]}\left\{| \phi ( t ) | \left[b_{1} \sum_{i=1}^{m} \mu_{i} \frac{1}{\rho^{\alpha+\beta+q-2} \Gamma(\alpha+\beta+q-1)}\right.\right. \\
& \cdot \int_{0}^{\xi_{i}}\left(\xi_{i}^{\rho}-s^{\rho}\right)^{\alpha+\beta+q-2} s^{\rho-1}|g(s, y(s))-g(s, \tilde{y}(s))| d s \\
& +b_{2} \sum_{i=1}^{p} r_{i} \frac{1}{\rho^{\alpha+\beta-2} \Gamma(\alpha+\beta-1)} \\
& \cdot \int_{0}^{\eta_{i}}\left(\eta_{i}^{\rho}-s^{\rho}\right)^{\alpha+\beta-2} s^{\rho-1}|g(s, y(s))-g(s, \tilde{y}(s))| d s \\
& -\frac{1}{\rho^{\alpha+\beta-2} \Gamma(\alpha+\beta-1)} \int_{0}^{1}\left(1-s^{\rho}\right)^{\alpha+\beta-2} s^{\rho-1} \mid g(s, y(s)) \\
& -g(s, \tilde{y}(s)) \mid d s]+\frac{1}{\left|\psi_{2}\right|} \\
& \cdot\left[a_{1} \sum_{i=1}^{m} \sigma_{i} \frac{1}{\rho^{\alpha+\beta+q-1} \Gamma(\alpha+\beta+q)}\right. \\
& \cdot \int_{0}^{\xi_{i}}\left(\xi_{i}^{\rho}-s^{\rho}\right)^{\alpha+\beta+q-1} s^{\rho-1}|g(s, y(s))-g(s, \tilde{y}(s))| d s \\
& +a_{2} \sum_{i=1}^{p} \delta_{i} \frac{1}{\rho^{\alpha+\beta-1} \Gamma(\alpha+\beta)} \\
& \left.\cdot \int_{0}^{\eta_{i}}\left(\eta_{i}^{\rho}-s^{\rho}\right)^{\alpha+\beta-1} s^{\rho-1}|g(s, y(s))-g(s, \tilde{y}(s))| d s\right\} .
\end{aligned}
$$

Therefore,

$$
\begin{aligned}
\left\|\mathscr{F}_{2} y-\mathscr{F}_{2} \tilde{y}\right\| \leq & \Omega\|y-\tilde{y}\|\left[\| \phi \| \left(b_{1} \sum_{i=1}^{m} \mu_{i} \frac{\left(\xi_{i}\right)^{\rho(\alpha+\beta+q-1)}}{\rho^{\alpha+\beta+q-1} \Gamma(\alpha+\beta+q)}\right.\right. \\
& \left.+\frac{1}{\rho^{\alpha+\beta-1} \Gamma(\alpha+\beta)}\left[b_{2} \sum_{i=1}^{p} r_{i}\left(\eta_{i}\right)^{\rho(\alpha+\beta-1)}-1\right]\right) \\
& +\frac{1}{\left|\psi_{2}\right|}\left(a_{1} \sum_{i=1}^{m} \sigma_{i} \frac{\left(\xi_{i}\right)^{\rho(\alpha+\beta+q)}}{\rho^{\alpha+\beta+q} \Gamma(\alpha+\beta+q+1)}\right. \\
& \left.\left.+a_{2} \sum_{i=1}^{p} \delta_{i} \frac{\left(\eta_{i}\right)^{\rho(\alpha+\beta)}}{\rho^{\alpha+\beta} \Gamma(\alpha+\beta+1)}\right)\right] \\
\leq & \Omega\|y-\tilde{y}\| \psi_{*} .
\end{aligned}
$$

By using (S1) and (48), $\mathscr{F}_{2}$ is a contraction. Since $g$ is continuous, we have $\mathscr{F}_{1}$ which is continuous. Now,

$$
\begin{aligned}
\left\|\mathscr{F}_{1} y\right\| & \leq \sup _{t \in[0,1]}\left|\frac{1}{\rho^{\alpha+\beta-1} \Gamma(\alpha+\beta)} \int_{0}^{t}\left(t^{\rho}-s^{\rho}\right)^{\alpha+\beta-1} s^{\rho-1} g(s, y(s)) d s\right| \\
& \leq \sup _{t \in[0,1]}\left\{\frac{1}{\rho^{\alpha+\beta-1} \Gamma(\alpha+\beta)} \int_{0}^{t}\left(t^{\rho}-s^{\rho}\right)^{\alpha+\beta-1} s^{\rho-1}|g(s, y(s))| d s\right\} \\
& \leq\|\zeta\|\left(\frac{1}{\rho^{\alpha+\beta} \Gamma(\alpha+\beta+1)}\right) .
\end{aligned}
$$


Thus, $\mathscr{F}_{1}$ is uniformly bounded on $B_{r^{*}}$. Next, for $t_{1}, t_{2}$ $\in[0,1]$ with $0<t_{1}<t_{2}<1$, we obtain

$$
\begin{aligned}
\left\|\left(\mathscr{F}_{1} y\right)\left(t_{2}\right)-\left(\mathscr{F}_{1} y\right)\left(t_{1}\right)\right\| \leq & \frac{1}{\rho^{\alpha+\beta-1} \Gamma(\alpha+\beta)} \\
& \cdot\left[\int_{0}^{t_{2}}\left(t_{2}^{\rho}-s^{\rho}\right)^{\alpha+\beta-1} s^{\rho-1}|g(s, y(s))| d s\right. \\
& \left.-\int_{0}^{t_{1}}\left(t_{1}^{\rho}-s^{\rho}\right)^{\alpha+\beta-1} s^{\rho-1}|g(s, y(s))| d s\right] \\
\leq & \frac{v \rho^{1-(\alpha+\beta)}}{\rho(\alpha+\beta) \Gamma(\alpha+\beta)}\left[2\left(t_{2}^{\rho}-t_{1}^{\rho}\right)^{\alpha+\beta}\right. \\
& \left.+\left(t_{2}^{\rho(\alpha+\beta)}-t_{1}^{\rho(\alpha+\beta)}\right)\right] \\
\leq & \frac{v}{\rho^{(\alpha+\beta)} \Gamma(\alpha+\beta+1)}\left[2\left(t_{2}^{\rho}-t_{1}^{\rho}\right)^{\alpha+\beta}\right. \\
& \left.+\left(t_{2}^{\rho(\alpha+\beta)}-t_{1}^{\rho(\alpha+\beta)}\right)\right],
\end{aligned}
$$

which tends to zero as $\left(t_{2}-t_{1}\right) \longrightarrow 0$ independent of $y$. Thus, $\mathscr{F}_{1}$ is equicontinuous. Hence, $\mathscr{F}_{1}$ is relatively compact on $B_{r^{*}}$ . Then, all assumptions of Krasnoselskii's fixed point theorem are satisfied, so the boundary value problem (1) has at least one solution on $[0,1]$.

The following example illustrates our main results.

\section{Example 1.}

$$
\left\{\begin{array}{l}
{ }^{R L} D_{0^{+}}^{1 / 2,1 / 6}\left({ }^{C} D_{0+}^{1 / 4,1 / 6} y(t)\right)=g(t, y(t)), 0<\alpha, \beta \leq 1, \\
y(0)=\frac{1}{7} \sum_{i=1}^{m} I^{3 / 4,1 / 6} y(\xi i)+\frac{2}{7} \sum_{i=1}^{1 / 6} \delta_{i} y(\eta i), \\
\gamma y(1)=\frac{3}{7} \sum_{i=1}^{2} \mu_{i} I^{3 / 4,1 / 6} \gamma y(\xi i)+\frac{4}{7} \sum_{i=1}^{2} r_{i} \gamma y(\eta i),
\end{array}\right.
$$

where $\xi_{1}=1 / 5, \xi_{2}=1 / 9, \eta_{1}=1 / 8, \eta_{2}=1 / 8, \delta_{1}=3 / 8, \delta_{2}=5 / 8$, $r_{1}=3 / 10, r_{2}=7 / 10, \sigma_{1}=4 / 9, \sigma_{2}=5 / 9, \mu_{1}=1 / 11, \mu_{1}=2 / 11$, $\left(\gamma=t^{1-\rho}(d / d t)\right)$, and $0<q<1$.

Let the function $g$ be defined by

$$
\begin{aligned}
g(t, y)= & \frac{\tan ^{-1} y}{10 \sqrt{\cos t+16}}+\left(\frac{2-t}{30}\right)\left(\frac{5|y|}{5|y|+1}\right) \\
& +\frac{e^{-t}}{20} \sqrt{\cos t+3}
\end{aligned}
$$

Using the given data, we find $\psi_{1}=0.1089838156, \psi_{2}=$ $0.2461605410, \quad \psi_{3}=1.807429635$, and $\phi=75.80569440$. Choose $\mathscr{G}(u)=|u|^{\alpha} / \alpha, \alpha>0$, with $\alpha=2$; then, $\mathscr{G}$ is an $N$-function and satisfies

$$
\int_{0}^{1} \mathscr{G}(|g(s, u(s))|) d s<\infty,
$$

which shows that $\mathscr{G}$ belongs to an Orlicz space $L_{\mathscr{G}}([0,1])$. We have

$$
\begin{aligned}
|g(t, y)-g(t, \tilde{y})|= & \mid\left(\frac{\tan ^{-1} y}{10 \sqrt{\cos t+16}}+\left(\frac{2-t}{30}\right)\left(\frac{5|y|}{5|y|+1}\right)\right. \\
& \left.+\frac{e^{-t}}{20} \sqrt{\cos t+3}\right)-\left(\frac{\tan ^{-1} \tilde{y}}{10 \sqrt{\cos t+16}}\right. \\
& \left.+\left(\frac{2-t}{30}\right)\left(\frac{5|\tilde{y}|}{5|\tilde{y}|+1}\right)+\frac{e^{-t}}{20} \sqrt{\cos t+3}\right) \mid \\
\leq & \frac{1}{10 \sqrt{\cos t+16}}\left|\tan ^{-1} y-\tan ^{-1} \tilde{y}\right| \\
& +\left(\frac{2-t}{30}\right)\left|\frac{5|y|}{5|y|+1}-\frac{5|\tilde{y}|}{5|\tilde{y}|+1}\right| .
\end{aligned}
$$

Consequently,

$$
\begin{aligned}
&|g(t, y)-g(t, \tilde{y})| \leq \frac{1}{40}|y-\tilde{y}|+\frac{1}{15}|y-\tilde{y}| \\
& \leq \frac{1}{40}|y-\tilde{y}|+\frac{1}{40}|y-\tilde{y}| \\
&=\frac{1}{20}|y-\tilde{y}| \leq \frac{1}{20}\|y-\tilde{y}\|, \\
&|g(t, y)-g(t, 0)| \leq \frac{1}{20}\|y\|,
\end{aligned}
$$

which shows the authenticity of (S1) with $\Omega \psi=0.8902242605$ $<1$; then, Theorem 14 can be illustrated and problem (56) has a unique solution on $[0,1]$. Furthermore,

$$
\mathrm{g}(t, y(t)) \leq v(t),
$$

with $\quad v(t)=\Pi /(20 \sqrt{\cos t+16})+(2-t) / 30+\left(e^{-t} / 20\right)$ $\sqrt{\cos t+3}$ and $\|v(t)\|=0.2$.

Since the condition (S2) holds and by the conditions of Theorem 15, problem (56) has at least one solution on $[0,1]$.

\section{Ulam Stability}

In this section, we establish the criteria of Ulam stability of problem (1) by means of its equivalent integral equation

$$
\begin{aligned}
w(t)= & \frac{1}{\rho^{\alpha+\beta-1} \Gamma(\alpha+\beta)} \int_{0}^{t}\left(t^{\rho}-s^{\rho}\right)^{\alpha+\beta-1} s^{\rho-1} g(s, w(s)) d s \\
& +\phi(t)\left[b_{1} \sum_{i=1}^{m} \mu_{i} \frac{1}{\rho^{\alpha+\beta+q-2} \Gamma(\alpha+\beta+q-1)}\right. \\
& \cdot \int_{0}^{\xi_{i}}\left(\xi_{i}^{\rho}-s^{\rho}\right)^{\alpha+\beta+q-2} s^{\rho-1} g(s, w(s)) d s \\
& +b_{2} \sum_{i=1}^{p} r_{i} \frac{1}{\rho^{\alpha+\beta-2} \Gamma(\alpha+\beta-1)}
\end{aligned}
$$




$$
\begin{aligned}
& \cdot \int_{0}^{\eta_{i}}\left(\eta_{i}^{\rho}-s^{\rho}\right)^{\alpha+\beta-2} s^{\rho-1} g(s, w(s)) d s \\
& \left.-\frac{1}{\rho^{\alpha+\beta-2} \Gamma(\alpha+\beta-1)} \int_{0}^{1}\left(1-s^{\rho}\right)^{\alpha+\beta-2} s^{\rho-1} g(s, w(s)) d s\right] \\
& +\frac{1}{\psi_{2}}\left[a_{1} \sum_{i=1}^{m} \sigma_{i} \frac{1}{\rho^{\alpha+\beta+q-1} \Gamma(\alpha+\beta+q)}\right. \\
& \cdot \int_{0}^{\xi_{i}}\left(\xi_{i}^{\rho}-s^{\rho}\right)^{\alpha+\beta+q-1} s^{\rho-1} g(s, w(s)) d s \\
& +a_{2} \sum_{i=1}^{p} \delta_{i} \frac{1}{\rho^{\alpha+\beta-1} \Gamma(\alpha+\beta)} \\
& \left.\cdot \int_{0}^{\eta_{i}}\left(\eta_{i}^{\rho}-s^{\rho}\right)^{\alpha+\beta-1} s^{\rho-1} g(s, w(s)) d s\right]
\end{aligned}
$$

where $w \in Y$ and $g:[0,1] \times \mathbb{R} \longrightarrow \mathbb{R}$ is a continuous function. We define a continuous nonlinear operator $\Lambda: Y$ $\longrightarrow Y$

$$
\Lambda w(t)={ }^{\mathrm{RL}} D_{0^{+}}^{\alpha, \rho}\left({ }^{\mathrm{C}} D_{0^{+}}^{\beta, \rho} w(t)\right)-g(t, w(t))
$$

Now, we recall some basic concepts of the Ulam stability. For more details, one can see [24-27].

Definition 17. GBVP is said to be Ulam-Hyers stable if there exists a real number $k>0$ such that for each $\varepsilon>0$ and for each solution $w \in Y$ of ((1)) satisfying the inequality

$$
\|\Lambda w(t)\| \leq \varepsilon, \quad t \in[0,1],
$$

there exists a solution $y \in Y$ of GBVP satisfying the inequality

$$
\|y-w\| \leq k \varepsilon_{*}, \quad t \in[0,1]
$$

where $\varepsilon_{*}$ is a positive real number depending on $\varepsilon$.

Definition 18. GBVP is generalized Ulam-Hyers stable if there exists $\tau \in C\left(\mathbb{R}^{+}, \mathbb{R}^{+}\right)$such that for each solution $w \in Y$ of $G B V P$, there exists a solution $y \in Y$ of GBVP with

$$
|y(t)-w(t)| \leq \tau(\varepsilon), \quad t \in[0,1] .
$$

Definition 19. GBVP is Ulam-Hyers Rassias stable with respect to $\varsigma \in C\left([0,1], \mathbb{R}^{+}\right)$if there exists a real number $k>0$ such that for each $\varepsilon>0$ and for each solution $w \in Y$ of $G B V P$

$$
|\Lambda w(t)| \leq \varepsilon \varsigma(t), \quad t \in[0,1],
$$

we can find a solution $y \in Y$ of GBVP satisfying the inequality

$$
|y(t)-w(t)| \leq k \varepsilon_{*} \varsigma(t), \quad t \in[0,1]
$$

where $\varepsilon_{*}$ is a positive real number depending on $\varepsilon$.
Theorem 20. Assume that the condition (S1) and $\Omega \psi<1$ hold. Then, the GBVP satisfies both Ulam-Hyers and generalized Ulam-Hyers stability criteria.

Proof. We know that $y \in Y$ is a unique solution of GBVP as in Theorem 14. Let $w \in Y$ be another solution of GBVP satisfying (64). Observe that the operator $\mathscr{F}$ and $\mathscr{F}-I$ are equivalent for every solution $w \in Y$ (given by (62)) of GBVP. Therefore, by the fixed point property of operator $\mathscr{F}$, we have

$$
\begin{aligned}
|w(t)-y(t)| & =|w(t)-\mathscr{F} w(t)+\mathscr{F} w(t)-\mathscr{F} y(t)| \\
& \leq|\mathscr{F} y(t)-\mathscr{F} w(t)|+|\mathscr{F} w(t)-w(t)| \\
& \leq \Omega \psi|w-y|+\varepsilon,
\end{aligned}
$$

where $\varepsilon>0$ and $\Omega \psi<1$. Taking the norm of (69) for $t \in[0,1]$ and solving for $\|w-y\|$, we obtain

$$
\|w-y\| \leq \frac{\varepsilon}{1-\Omega \psi}
$$

If we let $\varepsilon_{*}=\varepsilon /(1-\Omega \psi)$ and take $k=1$, then the UlamHyers stability condition is satisfied. More generally, defining $\tau(\varepsilon)=\varepsilon /(1-m \psi)$, the generalized Ulam-Hyers stability condition is also satisfied.

Theorem 21. Assume that (S1) and $\Omega \psi<1$ hold and there exists a function $\varsigma \in C\left([0,1], \mathbb{R}^{+}\right)$satisfying condition $((67))$. Then, the problem GBVP is Ulam-Hyers Rassias stable with respect to $\varsigma$.

Proof. Following the idea of the proof of Theorem 19, we can obtain that

$$
\|y-w\| \leq \varepsilon_{*} \varsigma(t)
$$

with $\varepsilon_{*}=\varepsilon /(1-\Omega \psi)$.

\section{Existence Results for Multivalued Maps}

In this section, we extend the results to cover the multivalued case. We recall some basic definitions on multivalued maps. For more details, one can see [28].

For a normed space $(X,\|\|$.$) , let \mathscr{P}_{\mathrm{cl}}(X)=\{y \in \mathscr{P}(x) ; y$ is closed $\}, \mathscr{P}_{\mathrm{b}}(X)=\{y \in \mathscr{P}(x) ; y$ is bounded $\}, \mathscr{P}_{\mathrm{cp}}(X)=\{y$ $\in \mathscr{P}(x) ; y$ is compact $\}$, and $\mathscr{P}_{\mathrm{cp}, \mathrm{c}}(X)=\{y \in \mathscr{P}(x) ; y$ is compact and convex $\}$. A multivalued map $G: X \longrightarrow \mathscr{P}(X)$ is convex (closed) valued if $G(x)$ is convex (closed) for all $x$ $\in X$. The map $G$ is bounded on bounded sets if $G(\mathbb{B})=$ $\cup_{x \in \mathbb{B}} G(x)$ is bounded in $X$ for all $\mathbb{B} \in \mathscr{P}_{b}(X)$, i.e.,

$$
\sup _{x \in \mathbb{B}}\{\sup \{|y|: y \in G(x)\}\}<\infty .
$$

$G$ is called upper semicontinuous (u.s.c.) on $X$ if for each $x_{0} \in X$, the set $G\left(x_{0}\right)$ is a nonempty closed subset of $X$, and if for each open set $N$ of $X$ containing $G\left(x_{0}\right)$, there exists an open neighbourhood $\mathcal{N}_{0}$ of $x_{0}$ such that $G\left(\mathcal{N}_{0}\right) \subseteq N$. $G$ is said to be completely continuous if $G(\mathbb{B})$ is relatively 
compact for every $\mathbb{B} \in \mathscr{P}_{b}(X)$. If the multivalued map $G$ is completely continuous with nonempty compact values, then $G$ is u.s.c. if and only if $G$ has a closed graph, i.e.,

$$
\begin{gathered}
x_{n} \longrightarrow x_{*}, \\
y_{n} \longrightarrow y_{*}, \\
y_{n} \in G\left(x_{n}\right) \text { imply } y_{*} \in G\left(x_{*}\right) .
\end{gathered}
$$

$G$ has a fixed point if there is $x \in X$ such that $x \in G(x)$. The fixed point set of multivalued operator $G$ will be denoted by Fix $G$. A multivalued map $G:[0,1] \longrightarrow \mathscr{P}_{\mathrm{cl}}(\mathbb{R})$ is said to be measurable if for every $y \in \mathbb{R}$, the function

$$
t \longrightarrow(y, G(t))=\inf \{|y-z|: z \in G(t)\}
$$

is measurable.

Definition 22. A multivalued map $F:[0,1] \times \mathbb{R} \longrightarrow \mathscr{P}(\mathbb{R})$ is said to be Carathéodory if

(i) $t \longrightarrow F(t, x)$ is measurable for each $x \in \mathbb{R}$

(ii) $x \longrightarrow F(t, x)$ is upper semicontinuous for almost all $t \in[0,1]$. Further, a Carathéodory function $F$ is called $L^{1}$ - Carathéodory if

(iii) for each $\alpha>0$, there exist $\varphi_{\alpha} \in L^{1}\left([0,1], \mathbb{R}^{+}\right)$such that

$$
\|F(t, x)\|=\sup \{|v|: v \in F(t, x)\} \leq \varphi_{\alpha}(t),
$$

for all $\|x\|_{\infty} \leq \alpha$ and for a.e. $t \in[0,1]$

For each $y \in C([0,1], \mathbb{R})$, the set of selections of $F$ is defined by

$$
S_{F, y}:=\left\{v \in L^{1}\left([0,1], \mathbb{R}^{+}\right): v(t) \in F(t, y(t)) \text { for a.e.t } \in[0,1]\right\} \text {. }
$$

Lemma 23. Let $X$ be a Banach space. Let $F:[0,1] \times \mathbb{R} \longrightarrow$ $\mathscr{P}_{c p, c}(X)$ be an $L^{1}$ - Carathéodory multivalued map and let $\Theta$ be a linear continuous mapping from $L^{1}([0,1], X)$ to $C([0$, 1], X). Then, the operator

$$
\begin{aligned}
\Theta \circ S_{F}: & C([0,1], X) \longrightarrow \mathscr{P}_{c p, c}(C([0,1], X)), \\
x & \mapsto\left(\Theta \circ S_{F}\right)(x)=\Theta\left(S_{F, x}\right)
\end{aligned}
$$

is a closed graph operator in $C([0,1], X) \times C([0,1], X)$.

Definition 24. A function $y \in C([0,1], \mathbb{R})$ is called a solution of problem ((2)) if we can find a function $g \in L^{1}([0,1], \mathbb{R})$ with $g$ $(t) \in G(t, y)$ a.e.on $[0,1]$ such that $y(0)=a_{1} \sum_{i=1}^{m} \sigma_{i} I_{0^{+}}^{q, \rho} y\left(\xi_{i}\right)+$ $a_{2} \sum_{i=1}^{p} \delta_{i} y\left(\eta_{i}\right), \gamma y(1)=b_{1} \sum_{i=1}^{m} \mu_{i} I_{0^{+}}^{q, \rho} \gamma y\left(\xi_{i}\right)+b_{2} \sum_{i=1}^{p} r_{i} \gamma y\left(\eta_{i}\right)$, and

$$
\begin{aligned}
y(t)= & I_{0^{+}}^{\alpha+\beta, \rho} g(t)+\phi(t)\left[b_{1} \sum_{i=1}^{m} \mu_{i} I_{0^{+}}^{\alpha+\beta+q-1, \rho} g\left(\xi_{i}\right)\right. \\
& \left.+b_{2} \sum_{i=1}^{p} r_{i} I_{0^{+}}^{\alpha+\beta-1, \rho} g\left(\eta_{i}\right)-I_{0^{+}}^{\alpha+\beta-1, \rho} g(1)\right] \\
& +\frac{1}{\psi_{2}}\left[a_{1} \sum_{i=1}^{m} \sigma_{i} I_{0^{+}}^{\alpha+\beta+q, \rho} g\left(\xi_{i}\right)+a_{2} \sum_{i=1}^{p} \delta_{i} I_{0^{+}}^{\alpha+\beta, \rho} g\left(\eta_{i}\right)\right] .
\end{aligned}
$$

4.1. The Upper Semicontinuous Case. To prove the existence of solutions for (2), we will apply Leray-Schauder nonlinear alternative for multivalued maps [29].

Theorem 25. Assume that

$$
G:[0,1] \times \mathbb{R} \longrightarrow \mathscr{P}_{c p, c}(\mathbb{R}) \text { is } L^{1}-\text { Caratheodory }
$$
where

$$
\mathscr{P}_{c p, c}(\mathbb{R})=\{\gamma \in \mathscr{P}(\mathbb{R}): \gamma \text { is compact and convex }\}
$$

(H2) there exists a continuous nondecreasing function $\omega$ $:[0, \infty) \longrightarrow(0, \infty)$ and a function $P \in C\left([0,1], \mathbb{R}^{+}\right)$such that

$$
\|g(t, y)\|_{\mathscr{P}}=\sup \{|y|: y \in g(t, y)\} \leq P(t) \omega(\|y\|)
$$

for all $(t, y) \in[0,1] \times \mathbb{R}$.

(H3) there exists a number $L>0$ such that

$$
\frac{L}{\|p\| \omega(L) \psi}>1 \text {, }
$$

where $\psi$ is given in (39).

Then, the B.V.P has at least one solution on $[0,1]$.

Proof. Let the operator $\tilde{\mathscr{F}}: C([0,1], \mathbb{R}) \longrightarrow \mathscr{P}(C([0,1], \mathbb{R})$ be defined by

$$
\begin{aligned}
& \tilde{\mathscr{F}}(y)=\left\{\begin{array}{c}
h(t) \in C([0,1], \mathbb{R}): \\
h(t)=\left\{\frac{1}{\rho^{\alpha+\beta-1} \Gamma(\alpha+\beta)} \int_{0}^{t}\left(t^{\rho}-s^{\rho}\right)^{\alpha+\beta-1} s^{\rho-1} g(s) d s\right.
\end{array}\right. \\
& +\phi(t)\left[b_{1} \sum_{i=1}^{m} \mu_{i} \frac{1}{\rho^{\alpha+\beta+q-2} \Gamma(\alpha+\beta+q-1)}\right. \\
& \cdot \int_{0}^{\xi_{i}}\left(\xi_{i}^{\rho}-s^{\rho}\right)^{\alpha+\beta+q-2} s^{\rho-1} g(s) d s \\
& +b_{2} \sum_{i=1}^{p} r_{i} \frac{1}{\rho^{\alpha+\beta-2} \Gamma(\alpha+\beta-1)} \\
& \cdot \int_{0}^{\eta_{i}}\left(\eta_{i}^{\rho}-s^{\rho}\right)^{\alpha+\beta-2} s^{\rho-1} g(s) d s
\end{aligned}
$$




$$
\begin{aligned}
& \left.-\frac{1}{\rho^{\alpha+\beta-21} \Gamma(\alpha+\beta-1)} \int_{0}^{1}\left(1-s^{\rho}\right)^{\alpha+\beta-2} s^{\rho-1} g(s) d s\right] \\
& +\frac{1}{\psi_{2}}\left[a_{1} \sum_{i=1}^{m} \sigma_{i} \frac{1}{\rho^{\alpha+\beta+q-1} \Gamma(\alpha+\beta+q)}\right. \\
& \quad \cdot \int_{0}^{\xi_{i}}\left(\xi_{i}^{\rho}-s^{\rho}\right)^{\alpha+\beta+q-1} s^{\rho-1} g(s) d s \\
& +a_{2} \sum_{i=1}^{p} \delta_{i} \frac{1}{\rho^{\alpha+\beta-1} \Gamma(\alpha+\beta)} \\
& \left.\left.\quad \cdot \int_{0}^{\eta_{i}}\left(\eta_{i}^{\rho}-s^{\rho}\right)^{\alpha+\beta-1} s^{\rho-1} g(s) d s\right],\right\}
\end{aligned}
$$

or $g \in S_{G, y}$. It is clear the fixed points of $\tilde{\mathscr{F}}$ are the solutions of problem (2). So, we need to verify that the operator satisfies all the conditions of the Leray-Schauder nonlinear alternative. This will be done in five steps.

Step 1. $\tilde{\mathscr{F}}(y)$ is convex for all $y \in C([0,1], \mathbb{R})$. Indeed, if $h_{1}$ and $h_{2}$ belong to $\tilde{\mathscr{F}}(y)$, then there exist $g_{1}, g_{2} \in S_{G, y}$ such that

$S_{G, y}=\left\{g \in L^{1}\left([0,1], \mathbb{R}^{+}\right): g(t) \in G(t, y(t))\right.$ for a.e. $\left.t \in[0,1]\right\}$.

For each $t \in[0,1]$, we have

$$
\begin{aligned}
h_{i}(t)= & \frac{1}{\rho^{\alpha+\beta-1} \Gamma(\alpha+\beta)} \int_{0}^{t}\left(t^{\rho}-s^{\rho}\right)^{\alpha+\beta-1} s^{\rho-1} g_{i}(s) d s \\
& +\phi(t)\left[b_{1} \sum_{i=1}^{m} \mu_{i} \frac{1}{\rho^{\alpha+\beta+q-2} \Gamma(\alpha+\beta+q-1)}\right. \\
& \cdot \int_{0}^{\xi_{i}}\left(\xi_{i}^{\rho}-s^{\rho}\right)^{\alpha+\beta+q-2} s^{\rho-1} g_{i}(s) d s \\
& +b_{2} \sum_{i=1}^{p} r_{i} \frac{1}{\rho^{\alpha+\beta-2} \Gamma(\alpha+\beta-1)} \\
& \cdot \int_{0}^{\eta_{i}}\left(\eta_{i}^{\rho}-s^{\rho}\right)^{\alpha+\beta-2} s^{\rho-1} g_{i}(s) d s \\
& \left.-\frac{1}{\rho^{\alpha+\beta-21} \Gamma(\alpha+\beta-1)} \int_{0}^{1}\left(1-s^{\rho}\right)^{\alpha+\beta-2} s^{\rho-1} g_{i}(s) d s\right] \\
& +\frac{1}{\psi_{2}}\left[a_{1} \sum_{i=1}^{m} \sigma_{i} \frac{1}{\rho^{\alpha+\beta+q-1} \Gamma(\alpha+\beta+q)}\right. \\
& \cdot \int_{0}^{\xi_{i}}\left(\xi_{i}^{\rho}-s^{\rho}\right)^{\alpha+\beta+q-1} s^{\rho-1} g_{i}(s) d s \\
& \left.+a_{2} \sum_{i=1}^{p} \delta_{i} \frac{1}{\eta^{\alpha+\beta-1} \Gamma(\alpha+\beta)}\left(\eta_{i}^{\rho}-s^{\rho}\right)^{\alpha+\beta-1} s^{\rho-1} g_{i}(s) d s\right], \\
& \int_{0}(84)
\end{aligned}
$$

for $i=1,2$. Let $t \in[0,1]$ and $\theta \in(0,1)$, then

$$
\begin{aligned}
& {\left[\theta h_{1}+(1-\theta) h_{2}\right](t)=\frac{1}{\rho^{\alpha+\beta-1} \Gamma(\alpha+\beta)} \int_{0}^{t}\left(t^{\rho}-s^{\rho}\right)^{\alpha+\beta-1} s^{\rho-1}\left[\theta g_{1}(s)\right.} \\
& \left.+(1-\theta) g_{2}(s)\right] d s+\phi(t) \\
& \text {. }\left[b_{1} \sum_{i=1}^{m} \mu_{i} \frac{1}{\rho^{\alpha+\beta+q-2} \Gamma(\alpha+\beta+q-1)}\right. \\
& \cdot \int_{0}^{\xi_{i}}\left(\xi_{i}^{\rho}-s^{\rho}\right)^{\alpha+\beta+q-2} s^{\rho-1}\left[\theta g_{1}(s)\right. \\
& \left.+(1-\theta) g_{2}(s)\right] d s \\
& +b_{2} \sum_{i=1}^{p} r_{i} \frac{1}{\rho^{\alpha+\beta-2} \Gamma(\alpha+\beta-1)} \\
& \cdot \int_{0}^{\eta_{i}}\left(\eta_{i}^{\rho}-s^{\rho}\right)^{\alpha+\beta-2} s^{\rho-1}\left[\theta g_{1}(s)\right. \\
& \left.+(1-\theta) g_{2}(s)\right] d s \\
& -\frac{1}{\rho^{\alpha+\beta-2} \Gamma(\alpha+\beta-1)} \\
& \cdot \int_{0}^{1}\left(1-s^{\rho}\right)^{\alpha+\beta-2} s^{\rho-1}\left[\theta g_{1}(s)\right. \\
& \left.\left.+(1-\theta) g_{2}(s)\right] d s\right] \\
& +\frac{1}{\psi_{2}}\left[a_{1} \sum_{i=1}^{m} \sigma_{i} \frac{1}{\rho^{\alpha+\beta+q-1} \Gamma(\alpha+\beta+q)}\right. \\
& \cdot \int_{0}^{\xi_{i}}\left(\xi_{i}^{\rho}-s^{\rho}\right)^{\alpha+\beta+q-1} s^{\rho-1}\left[\theta g_{1}(s)\right. \\
& \left.+(1-\theta) g_{2}(s)\right] d s+a_{2} \sum_{i=1}^{p} \delta_{i} \frac{1}{\rho^{\alpha+\beta-1} \Gamma(\alpha+\beta)} \\
& \left.\cdot \int_{0}^{\eta_{i}}\left(\eta_{i}^{\rho}-s^{\rho}\right)^{\alpha+\beta-1} s^{\rho-1}\left[\theta g_{1}(s)+(1-\theta) g_{2}(s)\right] d s\right] \text {. }
\end{aligned}
$$

Since $G$ has convex values $\left(S_{G, y}\right.$ is convex), therefore, $\theta$ $h_{1}+(1-\theta) h_{2} \in \tilde{\mathscr{F}}(y)$.

Step 2. $\tilde{\mathscr{F}}(y)$ maps bounded sets into bounded sets in $C([0$, $1], \mathbb{R})$. Let $B_{\varepsilon}=\{y \in C([0,1], \mathbb{R}):\|y\| \leq \varepsilon\}$, where $\varepsilon$ is a positive number. Then, for each $h \in \tilde{\mathscr{F}}(y), y \in B_{\varepsilon}$, there exists $g \in S_{G, y}$ such that

$$
\begin{aligned}
h(t)= & \frac{1}{\rho^{\alpha+\beta-1} \Gamma(\alpha+\beta)} \int_{0}^{t}\left(t^{\rho}-s^{\rho}\right)^{\alpha+\beta-1} s^{\rho-1} g(s) d s \\
& +\phi(t)\left[b_{1} \sum_{i=1}^{m} \mu_{i} \frac{1}{\rho^{\alpha+\beta+q-2} \Gamma(\alpha+\beta+q-1)}\right. \\
& \cdot \int_{0}^{\xi_{i}}\left(\xi_{i}^{\rho}-s^{\rho}\right)^{\alpha+\beta+q-2} s^{\rho-1} g(s) d s \\
& +b_{2} \sum_{i=1}^{p} r_{i} \frac{1}{\rho^{\alpha+\beta-2} \Gamma(\alpha+\beta-1)} \\
& \cdot \int_{0}^{\eta_{i}}\left(\eta_{i}^{\rho}-s^{\rho}\right)^{\alpha+\beta-2} s^{\rho-1} g(s) d s
\end{aligned}
$$




$$
\begin{aligned}
& \left.-\frac{1}{\rho^{\alpha+\beta-2} \Gamma(\alpha+\beta-1)} \int_{0}^{1}\left(1-s^{\rho}\right)^{\alpha+\beta-2} s^{\rho-1} g(s) d s\right] \\
& +\frac{1}{\psi_{2}}\left[a_{1} \sum_{i=1}^{m} \sigma_{i} \frac{1}{\rho^{\alpha+\beta+q-1} \Gamma(\alpha+\beta+q)}\right. \\
& \cdot \int_{0}^{\xi_{i}}\left(\xi_{i}^{\rho}-s^{\rho}\right)^{\alpha+\beta+q-1} s^{\rho-1} g(s) d s \\
& \left.+a_{2} \sum_{i=1}^{p} \delta_{i} \frac{1}{\rho^{\alpha+\beta-1} \Gamma(\alpha+\beta)} \int_{0}^{\eta_{i}}\left(\eta_{i}^{\rho}-s^{\rho}\right)^{\alpha+\beta-1} s^{\rho-1} g(s) d s\right]
\end{aligned}
$$

For each $t \in[0,1]$, we find that

$$
\begin{aligned}
& |h(t)| \leq \frac{1}{\rho^{\alpha+\beta-1} \Gamma(\alpha+\beta)} \int_{0}^{t}\left(t^{\rho}-s^{\rho}\right)^{\alpha+\beta-1} s^{\rho-1}|g(s)| d s \\
& +|\phi(t)|\left[b_{1} \sum_{i=1}^{m} \mu_{i} \frac{1}{\rho^{\alpha+\beta+q-2} \Gamma(\alpha+\beta+q-1)}\right. \\
& \cdot \int_{0}^{\xi_{i}}\left(\xi_{i}^{\rho}-s^{\rho}\right)^{\alpha+\beta+q-2} s^{\rho-1}|g(s)| d s \\
& +b_{2} \sum_{i=1}^{p} r_{i} \frac{1}{\rho^{\alpha+\beta-2} \Gamma(\alpha+\beta-1)} \\
& \cdot \int_{0}^{\eta_{i}}\left(\eta_{i}^{\rho}-s^{\rho}\right)^{\alpha+\beta-2} s^{\rho-1}|g(s)| d s \\
& \left.-\frac{1}{\rho^{\alpha+\beta-2} \Gamma(\alpha+\beta-1)} \int_{0}^{1}\left(1-s^{\rho}\right)^{\alpha+\beta-2} s^{\rho-1}|g(s)| d s\right] \\
& +\frac{1}{\left|\psi_{2}\right|}\left[a_{1} \sum_{i=1}^{m} \sigma_{i} \frac{1}{\rho^{\alpha+\beta+q-1} \Gamma(\alpha+\beta+q)}\right. \\
& \cdot \int_{0}^{\xi_{i}}\left(\xi_{i}^{\rho}-s^{\rho}\right)^{\alpha+\beta+q-1} s^{\rho-1}|g(s)| d s \\
& \left.+a_{2} \sum_{i=1}^{p} \delta_{i} \frac{1}{\rho^{\alpha+\beta-1} \Gamma(\alpha+\beta)} \int_{0}^{\eta_{i}}\left(\eta_{i}^{\rho}-s^{\rho}\right)^{\alpha+\beta-1} s^{\rho-1}|g(s)| d s\right] \\
& \leq\|\mathscr{P}\| \omega(\|y\|)\left(\frac{1}{\rho^{\alpha+\beta} \Gamma(\alpha+\beta+1)}+\|\phi\|\right. \\
& {\left[b_{1} \sum_{i=1}^{m} \mu_{i} \frac{\left(\xi_{i}\right)^{\rho(\alpha+\beta+q-1)}}{\rho^{\alpha+\beta+q-1} \Gamma(\alpha+\beta+q)}\right.} \\
& \left.+\frac{1}{\rho^{\alpha+\beta-1} \Gamma(\alpha+\beta)}\left[b_{2} \sum_{i=1}^{p} r_{i}\left(\eta_{i}\right)^{\rho(\alpha+\beta-1)}-1\right]\right] \\
& +\frac{1}{\left|\psi_{2}\right|}\left[a_{1} \sum_{i=1}^{m} \sigma_{i} \frac{\left(\xi_{i}\right)^{\rho(\alpha+\beta+q)}}{\rho^{\alpha+\beta+q} \Gamma(\alpha+\beta+q+1)}\right. \\
& \left.\left.+a_{2} \sum_{i=1}^{p} \delta_{i} \frac{\left(\eta_{i}\right)^{\rho(\alpha+\beta)}}{\rho^{\alpha+\beta} \Gamma(\alpha+\beta+1)}\right]\right)
\end{aligned}
$$$$
\leq\|\mathscr{P}\| \omega(\|y\|) \psi \text {, }
$$

Step 3. $\tilde{F}(y)$ maps bounded sets into equicontinuous sets in $C([0,1], \mathbb{R})$. Let $y$ be any element in $B_{\varepsilon}$ and $t_{1}, t_{2} \in[0,1], t_{1}$ $<t_{2}$; then, by using $(\mathrm{H} 2)$, we get that for each $t \in[0,1]$, we find that

$$
\begin{aligned}
\left|h\left(t_{2}\right)-h\left(t_{1}\right)\right| \leq & p(t) \omega(\varepsilon)\left\{\frac { 1 } { \rho ^ { \alpha + \beta } \Gamma ( \alpha + \beta + 1 ) } \left[2\left(t_{2}^{\rho}-t_{1}^{\rho}\right)^{\alpha+\beta}\right.\right. \\
& \left.+\left(t_{2}^{\rho(\alpha+\beta)}-t_{1}^{\rho(\alpha+\beta)}\right)\right] \\
& +\frac{1}{\psi_{1}}\left[\frac{\Gamma(\alpha)\left|t^{\rho(\alpha+\beta-1)}-\tau^{\rho(\alpha+\beta-1)}\right|}{\rho^{\beta} \Gamma(\alpha+\beta)}\right] \\
& \cdot\left[b_{1} \sum_{i=1}^{m} \mu_{i} \frac{\left(\xi_{i}\right)^{\rho(\alpha+\beta+q-1)}}{\rho^{\alpha+\beta+q-1} \Gamma(\alpha+\beta+q)}\right. \\
& \left.+\frac{1}{\rho^{\alpha+\beta-1} \Gamma(\alpha+\beta)}\left[b_{2} \sum_{i=1}^{p} r_{i}\left(\eta_{i}\right)^{\rho(\alpha+\beta-1)}-1\right]\right] \\
& +\frac{1}{\left|\psi_{2}\right|}\left[a_{1} \sum_{i=1}^{m} \sigma_{i} \frac{\left(\xi_{i}\right)^{\rho(\alpha+\beta+q)}}{\rho^{\alpha+\beta+q} \Gamma(\alpha+\beta+q+1)}\right. \\
& \left.\left.+a_{2} \sum_{i=1}^{p} \delta_{i} \frac{\left(\eta_{i}\right)^{\rho(\alpha+\beta)}}{\rho^{\alpha+\beta} \Gamma(\alpha+\beta+1)}\right]\right\}
\end{aligned}
$$

It follows that the right-hand side tends to zero independently of $y \in B_{\varepsilon}$ as $\left(t_{2}-t_{1}\right) \longrightarrow 0$. Combining the outcomes of Steps 1-3 with the Arzela-Ascoli theorem, we conclude that $\tilde{\mathscr{F}}$ is completely continuous.

Step 4. $\tilde{\mathscr{F}}$ has a closed graph. Suppose that there is $y_{n} \longrightarrow$ $y_{*}, h_{n} \in \tilde{\mathscr{F}}\left(y_{n}\right)$, and $h_{n} \longrightarrow h_{*}$. Then, we have to establish that $h_{*} \in \tilde{\mathscr{F}}\left(y_{*}\right)$. Since $h_{n} \in \tilde{\mathscr{F}}\left(y_{n}\right)$, there exists $g_{n} \in S_{G, y_{n}}$ and therefore, for each $t \in[0,1]$, we get

$$
\begin{aligned}
h_{n}(t)= & \frac{1}{\rho^{\alpha+\beta-1} \Gamma(\alpha+\beta)} \int_{0}^{t}\left(t^{\rho}-s^{\rho}\right)^{\alpha+\beta-1} s^{\rho-1} g_{n}(s) d s \\
& +|\phi(t)|\left[b_{1} \sum_{i=1}^{m} \mu_{i} \frac{1}{\rho^{\alpha+\beta+q-2} \Gamma(\alpha+\beta+q-1)}\right. \\
& \cdot \int_{0}^{\xi_{i}}\left(\xi_{i}^{\rho}-s^{\rho}\right)^{\alpha+\beta+q-2} s^{\rho-1} g_{n}(s) d s \\
& +b_{2} \sum_{i=1}^{p} r_{i} \frac{1}{\rho^{\alpha+\beta-2} \Gamma(\alpha+\beta-1)} \int_{0}^{\eta_{i}}\left(\eta_{i}^{\rho}-s^{\rho}\right)^{\alpha+\beta-2} s^{\rho-1} g_{n}(s) d s \\
& \left.-\frac{1}{\rho^{\alpha+\beta-2} \Gamma(\alpha+\beta-1)} \int_{0}^{1}\left(1-s^{\rho}\right)^{\alpha+\beta-2} s^{\rho-1} g_{n}(s) d s\right] \\
& +\frac{1}{\left|\psi_{2}\right|}\left[a_{1} \sum_{i=1}^{m} \sigma_{i} \frac{1}{\rho^{\alpha+\beta+q-1} \Gamma(\alpha+\beta+q)}\right. \\
& \cdot \int_{0}^{\xi_{i}}\left(\xi_{i}^{\rho}-s^{\rho}\right)^{\alpha+\beta+q-1} s^{\rho-1} g_{n}(s) d s \\
& \left.+a_{2} \sum_{i=1}^{p} \delta_{i} \frac{1}{\rho^{\alpha+\beta-1} \Gamma(\alpha+\beta)} \int_{0}^{\eta_{i}}\left(\eta_{i}^{\rho}-s^{\rho}\right)^{\alpha+\beta-1} s^{\rho-1} g_{n}(s) d s\right] .
\end{aligned}
$$

which gives $\|h\| \leq\|\mathscr{P}\| \omega(\varepsilon) \psi$. 
Next, we show that there exists $g_{*} \in S_{G, y_{*}}$ such that for each $t \in[0,1]$,

$$
\begin{aligned}
h_{*}(t)= & \frac{1}{\rho^{\alpha+\beta-1} \Gamma(\alpha+\beta)} \int_{0}^{t}\left(t^{\rho}-s^{\rho}\right)^{\alpha+\beta-1} s^{\rho-1} g_{*}(s) d s \\
& +|\phi(t)|\left[b_{1} \sum_{i=1}^{m} \mu_{i} \frac{1}{\rho^{\alpha+\beta+q-2} \Gamma(\alpha+\beta+q-1)}\right. \\
& \cdot \int_{0}^{\xi_{i}}\left(\xi_{i}^{\rho}-s^{\rho}\right)^{\alpha+\beta+q-2} s^{\rho-1} g_{*}(s) d s \\
& +b_{2} \sum_{i=1}^{p} r_{i} \frac{1}{\rho^{\alpha+\beta-2} \Gamma(\alpha+\beta-1)} \\
& \cdot \int_{0}^{\eta_{i}}\left(\eta_{i}^{\rho}-s^{\rho}\right)^{\alpha+\beta-2} s^{\rho-1} g_{*}(s) d s \\
& \left.-\frac{1}{\rho^{\alpha+\beta-2} \Gamma(\alpha+\beta-1)} \int_{0}^{1}\left(1-s^{\rho}\right)^{\alpha+\beta-2} s^{\rho-1} g_{*}(s) d s\right] \\
& +\frac{1}{\left|\psi_{2}\right|}\left[a_{1} \sum_{i=1}^{m} \sigma_{i} \frac{1}{\rho^{\alpha+\beta+q-1} \Gamma(\alpha+\beta+q)}\right. \\
& \cdot \int_{0}^{\xi_{i}}\left(\xi_{i}^{\rho}-s^{\rho}\right)^{\alpha+\beta+q-1} s^{\rho-1} g_{*}(s) d s \\
& \left.+a_{2} \sum_{i=1}^{p} \delta_{i} \frac{1}{\rho^{\alpha+\beta-1} \Gamma(\alpha+\beta)} \int_{0}^{\eta_{i}}\left(\eta_{i}^{\rho}-s^{\rho}\right)^{\alpha+\beta-1} s^{\rho-1} g_{*}(s) d s\right] .
\end{aligned}
$$

Consider the continuous linear operator

$$
\Xi: L^{1}([0,1], \mathbb{R}) \longrightarrow C([0,1], \mathbb{R})
$$

given by

$$
\begin{gathered}
g \longrightarrow \Xi(g)(t)=\frac{1}{\rho^{\alpha+\beta-1} \Gamma(\alpha+\beta)} \int_{0}^{t}\left(t^{\rho}-s^{\rho}\right)^{\alpha+\beta-1} s^{\rho-1} g(s) d s \\
+|\phi(t)|\left[b_{1} \sum_{i=1}^{m} \mu_{i} \frac{1}{\rho^{\alpha+\beta+q-2} \Gamma(\alpha+\beta+q-1)}\right. \\
\cdot \int_{0}^{\xi_{i}}\left(\xi_{i}^{\rho}-s^{\rho}\right)^{\alpha+\beta+q-2} s^{\rho-1} g(s) d s \\
+b_{2} \sum_{i=1}^{p} r_{i} \frac{1}{\rho^{\alpha+\beta-2} \Gamma(\alpha+\beta-1)} \\
\cdot \int_{0}^{\eta_{i}}\left(\eta_{i}^{\rho}-s^{\rho}\right)^{\alpha+\beta-2} s^{\rho-1} g(s) d s \\
\left.-\frac{1}{\rho^{\alpha+\beta-2} \Gamma(\alpha+\beta-1)} \int_{0}^{1}\left(1-s^{\rho}\right)^{\alpha+\beta-2} s^{\rho-1} g(s) d s\right] \\
+\frac{1}{\left|\psi_{2}\right|}\left[a_{1} \sum_{i=1}^{m} \sigma_{i} \frac{1}{\rho^{\alpha+\beta+q-1} \Gamma(\alpha+\beta+q)}\right. \\
\cdot \int_{0}^{\xi_{i}}\left(\xi_{i}^{\rho}-s^{\rho}\right)^{\alpha+\beta+q-1} s^{\rho-1} g(s) d s
\end{gathered}
$$

$$
\begin{aligned}
+ & a_{2} \sum_{i=1}^{p} \delta_{i} \frac{1}{\rho^{\alpha+\beta-1} \Gamma(\alpha+\beta)} \\
& \left.\cdot \int_{0}^{\eta_{i}}\left(\eta_{i}^{\rho}-s^{\rho}\right)^{\alpha+\beta-1} s^{\rho-1} g(s) d s\right] .
\end{aligned}
$$

Then, we can get

$$
\begin{aligned}
\left\|h_{n}(t)-h_{*}(t)\right\| \leq & \frac{1}{\rho^{\alpha+\beta-1} \Gamma(\alpha+\beta)} \int_{0}^{t}\left(t^{\rho}-s^{\rho}\right)^{\alpha+\beta-1} s^{\rho-1}\left|g_{n}(s)-g_{*}(s)\right| d s \\
& +|\phi(t)|\left[b_{1} \sum_{i=1}^{m} \mu_{i} \frac{1}{\rho^{\alpha+\beta+q-2} \Gamma(\alpha+\beta+q-1)}\right. \\
& \cdot \int_{0}^{\xi_{i}}\left(\xi_{i}^{\rho}-s^{\rho}\right)^{\alpha+\beta+q-2} s^{\rho-1}\left|g_{n}(s)-g_{*}(s)\right| d s \\
& +b_{2} \sum_{i=1}^{p} r_{i} \frac{1}{\rho^{\alpha+\beta-2} \Gamma(\alpha+\beta-1)} \\
& \cdot \int_{0}^{\eta_{i}}\left(\eta_{i}^{\rho}-s^{\rho}\right)^{\alpha+\beta-2} s^{\rho-1}\left|g_{n}(s)-g_{*}(s)\right| d s \\
& -\frac{1}{\rho^{\alpha+\beta-2} \Gamma(\alpha+\beta-1)} \\
& \left.\cdot \int_{0}^{1}\left(1-s^{\rho}\right)^{\alpha+\beta-2} s^{\rho-1}\left|g_{n}(s)-g_{*}(s)\right| d s\right] \\
& +\frac{1}{\left|\psi_{2}\right|}\left[a_{1} \sum_{i=1}^{m} \sigma_{i} \frac{1}{\rho^{\alpha+\beta+q-1} \Gamma(\alpha+\beta+q)}\right. \\
& \cdot \int_{0}^{\xi_{i}}\left(\xi_{i}^{\rho}-s^{\rho}\right)^{\alpha+\beta+q-1} s^{\rho-1}\left|g_{n}(s)-g_{*}(s)\right| d s \\
& +a_{2} \sum_{i=1}^{p} \delta_{i} \frac{1}{\rho^{\alpha+\beta-1} \Gamma(\alpha+\beta)} \\
& \left.\cdot \int_{0}^{\eta_{i}}\left(\eta_{i}^{\rho}-s^{\rho}\right)^{\alpha+\beta-1} s^{\rho-1}\left|g_{n}(s)-g_{*}(s)\right| d s\right],
\end{aligned}
$$

for some $g_{*} \in S_{G, y_{*}}$. Notice that $\left\|h_{n}(t)-h_{*}(t)\right\| \longrightarrow 0$ as $n$ $\longrightarrow \infty$. So, we conclude that $\Xi \circ S_{G, y}$ is a closed graph operator. Then, $h_{n} \in \Xi\left(S_{G, y_{n}}\right) \longrightarrow h_{*} \in S_{G, y_{*}}$.

Step 5. There exists an open set $\mathscr{G} \subseteq C([0,1], \mathbb{R})$ with $y \notin \theta \tilde{F}$ (y) for any $\theta \in(0,1)$ and for each $y \in \partial \mathscr{G}$. Take $\theta \in(0,1)$. Let $y$ be a solution of (2); then, there exists $g \in L^{1}([0,1], \mathbb{R})$ with $g \in S_{G, y}$ such that for $t \in[0,1]$, we have

$$
\begin{aligned}
y(t)= & \frac{1}{\rho^{(\alpha+\beta)-1} \Gamma(\alpha+\beta)} \int_{0}^{t}\left(t^{\rho}-s^{\rho}\right)^{\alpha+\beta-1} s^{\rho-1} g(s) d s \\
& +\phi(t)\left[b_{1} \sum_{i=1}^{m} \mu_{i} \frac{1}{\rho^{(\alpha+\beta+q-1)-1} \Gamma(\alpha+\beta+q-1)}\right. \\
& \cdot \int_{0}^{\xi_{i}}\left(\xi_{i}^{\rho}-s^{\rho}\right)^{\alpha+\beta+q-2} s^{\rho-1} g(s) d s \\
& +b_{2} \sum_{i=1}^{p} r_{i} \frac{1}{\rho^{(\alpha+\beta-1)-1} \Gamma(\alpha+\beta-1)}
\end{aligned}
$$




$$
\begin{gathered}
\cdot \int_{0}^{\eta_{i}}\left(\eta_{i}^{\rho}-s^{\rho}\right)^{\alpha+\beta-2} s^{\rho-1} g(s) d s \\
\left.-\frac{1}{\rho^{(\alpha+\beta-1)-1} \Gamma(\alpha+\beta-1)} \int_{0}^{1}\left(1-s^{\rho}\right)^{\alpha+\beta-2} s^{\rho-1} g(s) d s\right] \\
+\frac{1}{\psi_{2}}\left[a_{1} \sum_{i=1}^{m} \sigma_{i} \frac{1}{\rho^{(\alpha+\beta+q)-1} \Gamma(\alpha+\beta+q)}\right. \\
+a_{2} \sum_{i=1}^{p} \delta_{i} \frac{1}{\rho^{(\alpha+\beta)-1} \Gamma(\alpha+\beta)} \int_{0}^{\xi_{i}}\left(\xi_{i}^{\rho}-s^{\rho}\right)^{\alpha+\beta+q-1} s^{\rho-1} g(s) d s
\end{gathered}
$$

Similar to the computation in Step 2, for each $t \in[0,1]$, we obtain

$$
|y(t)| \leq\|p\| \omega(\|y\|) \psi
$$

which implies

$$
\frac{\|y(t)\|}{\|p\| \omega(\|y\|) \psi}<1 .
$$

By (H3), there exists $L>0$ such that $\|y\| \neq L$. Define a set

$$
\mathscr{G}=\{y \in C([0,1], \mathbb{R}):\|y\|<L\} .
$$

Note that the operator $\tilde{\mathscr{F}}: \overline{\mathscr{G}} \longrightarrow \mathscr{P}(C[0,1], \mathbb{R})$ is a compact multivalued map, u.s.c. with convex closed values. With the given choice of $\mathscr{G}$, it is not possible to find $y \in \partial \mathscr{G}$ satisfying $y \in \theta \tilde{\mathscr{F}}(y)$ for some $\theta \in(0,1)$. Consequently, by the nonlinear alternative of the Leray-Schauder theorem, the operator $\tilde{\mathscr{F}}(y)$ has a fixed point $y \in \overline{\mathscr{G}}$, which is a solution of problem (2).

4.2. The Lipschitz Case. The following result is concerned with the nonconvex valued case of problem (2) and is based on Covitz and Nadler's fixed point theorem [30].

\section{Theorem 26. Assume that}

(H4) $G:[0,1] \times \mathbb{R} \longrightarrow \mathscr{P}_{c p}(\mathbb{R})$ is such that $G(., y):[0,1]$ $\longrightarrow \mathscr{P}_{c p}(\mathbb{R})$ is measurable for each $x \in \mathbb{R}$, where

$$
\mathscr{P}_{c p}(\mathbb{R})=\{\gamma \in \mathscr{P}(\mathbb{R}): \gamma \text { is compact and convex }\}
$$

(H5) $H^{-} d(G(t, y), G(t, \tilde{y})) \leq \omega(t)|y-\tilde{y}|$ for almost all $t \in[0,1]$ and $y, \tilde{y} \in \mathbb{R}$ with $\omega \in C\left([0,1], \mathbb{R}^{+}\right)$and $d(0, G(0$, $G(t, 0))) \leq \omega(t)$ for almost all $t \in[0,1]$.

Then, problem (81) has at least one solution on $[0,1]$ if

$$
\|\omega\| \psi<1
$$

Proof. Let us verify that the operator $\tilde{\mathscr{F}}: C([0,1], \mathbb{R}) \longrightarrow \mathscr{P}$
$(C[0,1], \mathbb{R})$ satisfies the hypothesis of the Covitz and Nadler fixed point theorem. We show that $\tilde{\mathscr{F}}(y)$ is nonempty and closed for every $\tilde{g} \in S_{G, y}$. Since the set-valued map $G(., y()$. is measurable (by Theorem III.6 in [28]), it admits a measurable selection $g:[0,1] \longrightarrow \mathbb{R}$, and we have

$$
|g(t)| \leq \omega(t)(1+|y(t)|),
$$

i.e., $g \in L^{1}([0,1], \mathbb{R})$, and hence, $G$ is integrably bounded. Therefor, $S_{G, y} \neq \phi$.

To prove that $\tilde{\mathscr{F}}(y)$ is closed for each $y \in C([0,1], \mathbb{R})$, let $\left\{h_{n}\right\}_{n \geq 0} \in \tilde{\mathscr{F}}(y)$ be such that $h_{n} \longrightarrow h$ as $n \longrightarrow \infty$ in $C([0,1]$, $\mathbb{R})$. Then, $h \in C([0,1], \mathbb{R})$ and we can find $g_{n} \in S_{G, y_{n}}$ such that for each $t \in[0,1]$,

$$
\begin{aligned}
h_{n}(t)= & \frac{1}{\rho^{\alpha+\beta-1} \Gamma(\alpha+\beta)} \int_{0}^{t}\left(t^{\rho}-s^{\rho}\right)^{\alpha+\beta-1} s^{\rho-1} g_{n}(s) d s \\
& +|\phi(t)|\left[b_{1} \sum_{i=1}^{m} \mu_{i} \frac{1}{\rho^{\alpha+\beta+q-2} \Gamma(\alpha+\beta+q-1)}\right. \\
& \cdot \int_{0}^{\xi_{i}}\left(\xi_{i}^{\rho}-s^{\rho}\right)^{\alpha+\beta+q-2} s^{\rho-1} g_{n}(s) d s \\
& +b_{2} \sum_{i=1}^{p} r_{i} \frac{1}{\rho^{\alpha+\beta-2} \Gamma(\alpha+\beta-1)} \\
& \cdot \int_{0}^{\eta_{i}}\left(\eta_{i}^{\rho}-s^{\rho}\right)^{\alpha+\beta-2} s^{\rho-1} g_{n}(s) d s \\
& \left.-\frac{1}{\rho^{\alpha+\beta-2} \Gamma(\alpha+\beta-1)} \int_{0}^{1}\left(1-s^{\rho}\right)^{\alpha+\beta-2} s^{\rho-1} g_{n}(s) d s\right] \\
& +\frac{1}{\left|\psi_{2}\right|}\left[a_{1} \sum_{i=1}^{m} \sigma_{i} \frac{1}{\rho^{\alpha+\beta+q-1} \Gamma(\alpha+\beta+q)}\right. \\
& \cdot \int_{0}^{\xi_{i}}\left(\xi_{i}^{\rho}-s^{\rho}\right)^{\alpha+\beta+q-1} s^{\rho-1} g_{n}(s) d s \\
& \left.+a_{2} \sum_{i=1}^{p} \delta_{i} \frac{1}{\rho^{\alpha+\beta-1} \Gamma(\alpha+\beta)} \int_{0}^{\eta_{i}}\left(\eta_{i}^{\rho}-s^{\rho}\right)^{\alpha+\beta-1} s^{\rho-1} g_{n}(s) d s\right] .
\end{aligned}
$$

As $G$ has compact values, we can pass onto a subsequence (if necessary) to obtain that $g_{n}$ converge to $g$ in $L^{1}([0,1], \mathbb{R})$. So $g \in S_{G, y}$. Then, for each $t \in[0,1]$, we have

$$
\begin{aligned}
h_{n}(t) \longrightarrow h(t) & =\frac{1}{\rho^{\alpha+\beta-1} \Gamma(\alpha+\beta)} \int_{0}^{t}\left(t^{\rho}-s^{\rho}\right)^{\alpha+\beta-1} s^{\rho-1} g(s) d s \\
+\phi(t) & {\left[b_{1} \sum_{i=1}^{m} \mu_{i} \frac{1}{\rho^{\alpha+\beta+q-2} \Gamma(\alpha+\beta+q-1)}\right.} \\
& \cdot \int_{0}^{\xi_{i}}\left(\xi_{i}^{\rho}-s^{\rho}\right)^{\alpha+\beta+q-2} s^{\rho-1} g(s) d s \\
& +b_{2} \sum_{i=1}^{p} r_{i} \frac{1}{\rho^{\alpha+\beta-2} \Gamma(\alpha+\beta-1)} \\
& \cdot \int_{0}^{\eta_{i}}\left(\eta_{i}^{\rho}-s^{\rho}\right)^{\alpha+\beta-2} s^{\rho-1} g(s) d s
\end{aligned}
$$




$$
\begin{aligned}
& \left.-\frac{1}{\rho^{\alpha+\beta-2} \Gamma(\alpha+\beta-1)} \int_{0}^{1}\left(1-s^{\rho}\right)^{\alpha+\beta-2} s^{\rho-1} g(s) d s\right] \\
& +\frac{1}{\psi_{2}}\left[a_{1} \sum_{i=1}^{m} \sigma_{i} \frac{1}{\rho^{\alpha+\beta+q-1} \Gamma(\alpha+\beta+q)}\right. \\
& \cdot \int_{0}^{\xi_{i}}\left(\xi_{i}^{\rho}-s^{\rho}\right)^{\alpha+\beta+q-1} s^{\rho-1} g(s) d s \\
& +a_{2} \sum_{i=1}^{p} \delta_{i} \frac{1}{\rho^{\alpha+\beta-1} \Gamma(\alpha+\beta)} \\
& \left.\cdot \int_{0}^{\eta_{i}}\left(\eta_{i}^{\rho}-s^{\rho}\right)^{\alpha+\beta-1} s^{\rho-1} g(s) d s\right] .
\end{aligned}
$$

which implies that $h \in \tilde{\mathscr{F}}(y)$. Now, we establish that there exists $0<\widehat{\theta}<1$, where it satisfies

$$
H_{d}(\tilde{\mathscr{F}}(y),
$$

$\tilde{\mathscr{F}}(\tilde{y})) \leq \widehat{\theta}\|y-\tilde{y}\| \quad$ for each $y, \tilde{y} \in C([0,1], \mathbb{R})$.

Let us take $y, \tilde{y} \in C([0,1], \mathbb{R})$ and $h_{1} \in \tilde{\mathscr{F}}(y)$. Then, there exists $\tilde{g}_{1} \in G(t, y(t))$ such that for all $t \in[0,1]$,

$$
\begin{aligned}
h_{1}(t)= & \frac{1}{\rho^{\alpha+\beta-1}} \Gamma(\alpha+\beta) \int_{0}^{t}\left(t^{\rho}-s^{\rho}\right)^{\alpha+\beta-1} s^{\rho-1} g_{1}(s) d s \\
& +\phi(t)\left[b_{1} \sum_{i=1}^{m} \mu_{i} \frac{1}{\rho^{\alpha+\beta+q-2} \Gamma(\alpha+\beta+q-1)}\right. \\
& \cdot \int_{0}^{\xi_{i}}\left(\xi_{i}^{\rho}-s^{\rho}\right)^{\alpha+\beta+q-2} s^{\rho-1} g_{1}(s) d s \\
& +b_{2} \sum_{i=1}^{p} r_{i} \frac{1}{\rho^{\alpha+\beta-2} \Gamma(\alpha+\beta-1)} \\
& \cdot \int_{0}^{\eta_{i}}\left(\eta_{i}^{\rho}-s^{\rho}\right)^{\alpha+\beta-2} s^{\rho-1} g_{1}(s) d s \\
& -\frac{1}{\rho^{\alpha+\beta-2} \Gamma(\alpha+\beta-1)} \\
& \left.\cdot \int_{0}^{1}\left(1-s^{\rho}\right)^{\alpha+\beta-2} s^{\rho-1} g_{1}(s) d s\right] \\
& +\frac{1}{\psi_{2}}\left[a_{1} \sum_{i=1}^{m} \sigma_{i} \frac{1}{\rho^{\alpha+\beta+q-1} \Gamma(\alpha+\beta+q)}\right. \\
& \cdot \int_{0}^{\xi_{i}}\left(\xi_{i}^{\rho}-s^{\rho}\right)^{\alpha+\beta+q-1} s^{\rho-1} g_{1}(s) d s \\
& +a_{2} \sum_{i=1}^{p} \delta_{i} \frac{1}{\rho^{\alpha+\beta-1} \Gamma(\alpha+\beta)} \\
& \left.\cdot \int_{0}^{\eta_{i}}\left(\eta_{i}^{\rho}-s^{\rho}\right)^{\alpha+\beta-1} s^{\rho-1} g_{1}(s) d s\right] .
\end{aligned}
$$

By (H5), we have

$$
H_{d}(G(t, y), G(t, \tilde{y}(t)) \leq \omega(t)|y(t)-\tilde{y}(t)| .
$$

So, there exists $\rho \in G(t, \tilde{y}(t))$ satisfying

$$
\left|g_{1}(t)-\rho\right| \leq \omega(t)|y(t)-\tilde{y}(t)|, \quad t \in[0,1] .
$$

Define $\mathrm{E}(t)=\left\{\rho \in \mathbb{R}:\left|g_{1}(t)-\rho\right| \leq \omega(t)|y(t)-\tilde{y}(t)|\right\}$. As the multivalued operator $\mathrm{E}(t) \cap G(t, \tilde{y}(t))$ is measurable, we can find a measurable selection $g_{2}(t)$ for $\mathrm{E}(t)$ (by Theorem III.2 in [28]). Thus, $g_{2}(t) \in G(t, \tilde{y}(t))$ and for each $t \in[0,1]$, we have

$$
\left|g_{1}(t)-g_{2}(t)\right| \leq \omega(t)|y-\tilde{y}| .
$$

For each $t \in[0,1]$, define

$$
\begin{aligned}
h_{2}(t)= & \frac{1}{\rho^{\alpha+\beta-1} \Gamma(\alpha+\beta)} \int_{0}^{t}\left(t^{\rho}-s^{\rho}\right)^{\alpha+\beta-1} s^{\rho-1} g_{2}(s) d s \\
& +\phi(t)\left[b_{1} \sum_{i=1}^{m} \mu_{i} \frac{1}{\rho^{\alpha+\beta+q-2} \Gamma(\alpha+\beta+q-1)}\right. \\
& \cdot \int_{0}^{\xi_{i}}\left(\xi_{i}^{\rho}-s^{\rho}\right)^{\alpha+\beta+q-2} s^{\rho-1} g_{2}(s) d s \\
& +b_{2} \sum_{i=1}^{p} r_{i} \frac{1}{\rho^{\alpha+\beta-2} \Gamma(\alpha+\beta-1)} \int_{0}^{\eta_{i}}\left(\eta_{i}^{\rho}-s^{\rho}\right)^{\alpha+\beta-2} s^{\rho-1} g_{2}(s) d s \\
& \left.-\frac{1}{\rho^{\alpha+\beta-2} \Gamma(\alpha+\beta-1)} \int_{0}^{1}\left(1-s^{\rho}\right)^{\alpha+\beta-2} s^{\rho-1} g_{2}(s) d s\right] \\
& +\frac{1}{\psi_{2}}\left[a_{1} \sum_{i=1}^{m} \sigma_{i} \frac{1}{\rho^{\alpha+\beta+q-1} \Gamma(\alpha+\beta+q)}\right. \\
& \cdot \int_{0}^{\xi_{i}}\left(\xi_{i}^{\rho}-s^{\rho}\right)^{\alpha+\beta+q-1} s^{\rho-1} g_{2}(s) d s \\
& \left.+a_{2} \sum_{i=1}^{p} \delta_{i} \frac{1}{\rho^{\alpha+\beta-1} \Gamma(\alpha+\beta)} \int_{0}^{\eta_{i}}\left(\eta_{i}^{\rho}-s^{\rho}\right)^{\alpha+\beta-1} s^{\rho-1} g_{2}(s) d s\right] .
\end{aligned}
$$

As a result, we get

$$
\begin{aligned}
&\left\|h_{1}(t)-h_{2}(t)\right\| \leq \frac{1}{\rho^{(\alpha+\beta)-1} \Gamma(\alpha+\beta)} \int_{0}^{t}\left(t^{\rho}-s^{\rho}\right)^{\alpha+\beta-1} s^{\rho-1}\left|g_{1}(s)-g_{2}(s)\right| d s \\
&+|\phi(t)|\left[b_{1} \sum_{i=1}^{m} \mu_{i} \frac{1}{\rho^{(\alpha+\beta+q-1)-1} \Gamma(\alpha+\beta+q-1)}\right. \\
& \cdot \int_{0}^{\xi_{i}}\left(\xi_{i}^{\rho}-s^{\rho}\right)^{\alpha+\beta+q-2} s^{\rho-1}\left|g_{1}(s)-g_{2}(s)\right| d s \\
& \quad+b_{2} \sum_{i=1}^{p} r_{i} \frac{1}{\rho^{(\alpha+\beta-1)-1} \Gamma(\alpha+\beta-1)} \\
& \cdot \int_{0}^{\eta_{i}}\left(\eta_{i}^{\rho}-s^{\rho}\right)^{\alpha+\beta-2} s^{\rho-1}\left|g_{1}(s)-g_{2}(s)\right| d s
\end{aligned}
$$




$$
\begin{gathered}
-\frac{1}{\rho^{(\alpha+\beta-1)-1} \Gamma(\alpha+\beta-1)} \\
\left.\cdot \int_{0}^{1}\left(1-s^{\rho}\right)^{\alpha+\beta-2} s^{\rho-1}\left|g_{1}(s)-g_{2}(s)\right| d s\right] \\
+\frac{1}{\left|\psi_{2}\right|}\left[a_{1} \sum_{i=1}^{m} \sigma_{i} \frac{1}{\rho^{(\alpha+\beta+q)-1} \Gamma(\alpha+\beta+q)}\right. \\
\cdot \int_{0}^{\xi_{i}}\left(\xi_{i}^{\rho}-s^{\rho}\right)^{\alpha+\beta+q-1} s^{\rho-1}\left|g_{1}(s)-g_{2}(s)\right| d s \\
\quad+a_{2} \sum_{i=1}^{p} \delta_{i} \frac{1}{\rho^{(\alpha+\beta)-1} \Gamma(\alpha+\beta)} \\
\left.\int_{0}^{\eta_{i}}\left(\eta_{i}^{\rho}-s^{\rho}\right)^{\alpha+\beta-1} s^{\rho-1}\left|g_{1}(s)-g_{2}(s)\right| d s\right] .
\end{gathered}
$$

Hence,

$$
\left|h_{1}(t)-h_{2}(t)\right| \leq\|\bowtie\| \psi\|y-\tilde{y}\|
$$

By interchange the roles of $y$ and $\tilde{y}$, we get

$$
H_{d}(\mathscr{F}(y)-\tilde{\mathscr{F}}(\tilde{y})) \leq\|\omega\| \psi\|y-\tilde{y}\|
$$

which implies that $\mathscr{F}$ is a contraction. Hence, by the conclusion of the Covitz and Nadler fixed point theorem, $\mathscr{F}$ has a fixed point.

\section{Data Availability}

No data were used to support this study.

\section{Conflicts of Interest}

The authors declare no conflict of interest.

\section{References}

[1] U. N. Katugampola, "New approach to a generalized fractional integral," Applied Mathematics and Computation, vol. 218, no. 3, pp. 860-865, 2011.

[2] U. N. Katugampola, "A new approach to generalized fractional derivatives," Bulltin of Mathematical Analysis and Applied, vol. 6, no. 4, pp. 1-15, 2014.

[3] F. Jarad, T. Abdeljawad, and J. Alzabut, "Generalized fractional derivatives generated by a class of local proportional derivatives," The European Physical Journal Special Topics, vol. 226, no. 16-18, pp. 3457-3471, 2017.

[4] F. Jarad, T. Abdeljawad, and D. Baleanu, "On the generalized fractional derivatives and their Caputo modification," Journal of Nonlinear Science and application, vol. 10, no. 5, pp. 26072619, 2017.

[5] Y. Y. Gambo, R. Ameen, F. Jarad, and T. Abdeljawad, "Existence and uniqueness of solutions to fractional differential equations in the frame of generalized Caputo fractional derivatives," Advances in Difference Equations, vol. 2018, no. 1, Article ID 134, 2018.
[6] A. Alsaedi, M. Alghanmi, B. Ahmad, and S. Ntouyas, "Generalized Liouville-Caputo fractional differential equations and inclusions with nonlocal generalized fractional integral and multipoint boundary conditions," Symmetry, vol. 10, no. 12, p. 667, 2018.

[7] A. Atangana and D. Baleanu, "New fractional derivatives with nonlocal and non-singular kernel: theory and application to heat transfer model," Thermal Science, vol. 20, no. 2, pp. 763-769, 2016.

[8] B. Ahmad and J. J. Nieto, "Existence results for a coupled system of nonlinear fractional differential equations with threepoint boundary conditions," Computers and Mathematics with Applications, vol. 58, no. 9, pp. 1838-1843, 2009.

[9] B. Ahmad and J. J. Nieto, "Existence of solutions for nonlocal boundary value problems of higher-order nonlinear fractional differential equations," Abstract and Applied Analysis, vol. 2009, 9 pages, 2009.

[10] B. Ahmad and J. J. Nieto, "Existence results for nonlinear boundary value problems of fractional integrodifferential equations with integral boundary conditions," Boundary Value Problems, vol. 2009, Article ID 708576, 11 pages, 2009.

[11] B. Ahmad and S. Sivasundaram, "Existence and uniqueness results for nonlinear boundary value problems of fractional differential equations with separated boundary conditions," Communications in Applied Analysis, vol. 13, no. 1, pp. 121128, 2009.

[12] Y. Ding, Z. Wang, and H. Ye, "Optimal control of a fractionalorder HIV-immune system with memory," IEEE Transactions on Control Systems Technology, vol. 20, no. 3, pp. 763-769, 2012.

[13] M. Javidi and B. Ahmad, "Dynamic analysis of time fractional order phytoplankton-toxic phytoplankton-zooplankton system," Ecological Modelling, vol. 318, pp. 8-18, 2015.

[14] K. S. Miller and B. Ross, An Introduction to the Fractional Calculus and Fractional Differential Equations, John Wiley, New York, 1993.

[15] I. Podlubny, Fractional Differential Equations, Academic Press, San Diego, 1999.

[16] A. A. Kilbas, H. M. Srivastava, and J. J. Trujillo, Theory and Applications of Fractional Differential Equations, North-Holland, Mathematical Studies, Elsevier, Amsterdam, 2006.

[17] S. G. Samko, A. A. Kilbas, and O. I. Marichev, Fractional Integrals and Derivatives: Theory and Applications, Gordon and Breach, Switzerland, 1993.

[18] Y. Cui, W. Ma, Q. Sun, and X. Su, "New uniqueness results for boundary value problem of fractional differential equation," Nonlinear Analysis: Modelling and Control, vol. 23, no. 1, pp. 31-39, 2018.

[19] B. Ahmad, M. Alghanmi, S. K. Ntouyas, and A. Alsaedi, "Fractional differential equations involving generalized derivative with Stieltjes and fractional integral boundary conditions," Applied Mathematics Letters, vol. 84, pp. 111117, 2018.

[20] B. Ahmad, A. Alsaedi, S. K. Ntouyas, and W. Shammakh, "On more general boundary value problems involving sequential fractional derivatives," Advances in Difference Equations, vol. 2019, Article ID 290, 2019.

[21] W. Shammakh and H. Z. Alzumi, "Existence results for nonlinear fractional boundary value problem involving generalized proportional derivative," Advances in Difference Equations, vol. 2019, no. 1, Article ID 94, 2019. 
[22] Z. Birnbaum and W. Orlicz, "Über die Verallgemeinerung des Begriffes der zueinander konjugierten Potenzen," Studia Mathematica, vol. 3, no. 1, pp. 1-67, 1931.

[23] D. R. Smart, Fixed Point Theorems, Cambridge University Press, Cambridge, 1980.

[24] D. H. Hyers, G. Isac, and T. M. Rassias, Stability of Fractional Equation in Several Variables, Progress in Nonlinear Differential Equations their Applications, vol. 34, Birkhauser, Boston, 1998.

[25] A. Alsaedi, M. Alsulami, R. P. Agarwal, and B. Ahmad, "Some new nonlinear second-order boundary value problems on an arbitrary domain," Advances in Difference Equations, vol. 2018, no. 227, 2018.

[26] R. Ameen, F. Jarad, and T. Abdeljawad, "Ulam stability for delay fractional differential equations with a generalized Caputo derivative," Univerzitet u Nišu, vol. 32, no. 15, pp. 5265-5274, 2018.

[27] U. Riaz, A. Zada, Z. Ali, Y. Cui, and J. Xu, “Analysis of coupled systems of implicit impulsive fractional differential equations involving Hadamard derivatives," Advances in Difference Equations, vol. 2019, no. 1, Article ID 226, 2019.

[28] C. Castaing and M. Valadier, "Convex Analysis and Measurable Multifunctions," in Lecture Notes in Mathematics, vol. 580, Spinger, Berlin, 1977.

[29] A. Grans and J. Dugundji, Fixed Point Theory, Springer, New York, 2005.

[30] H. Covitz and S. B. Nadler Jr., "Multi-valued contraction mappings in generalized metric spaces," Israel Journal Mathematics, vol. 8, no. 1, pp. 5-11, 1970. 